\title{
Roughness picture of friction in dry nanoscale contacts
}

\author{
Yifei Mo ${ }^{1}$ and Izabela Szlufarska ${ }^{1,2}$ \\ ${ }^{1}$ Materials Science Program, University of Wisconsin, Madison, Wisconsin 53706-1595, USA \\ ${ }^{2}$ Department of Materials Science \& Engineering, University of Wisconsin, Madison, Wisconsin 53706-1595, USA
}

(Received 17 August 2009; revised manuscript received 30 November 2009; published 7 January 2010)

\begin{abstract}
Large-scale molecular-dynamics simulations are performed to study friction in nanoscale single asperity contacts. The modeling system consists of a tip made of H-terminated diamond-like carbon and an $\mathrm{H}$-terminated diamond sample. Simulations are carried out using a reactive bond-order interatomic potential integrated with dispersive interactions. A quantitative agreement in contact pressures and shear strengths is achieved between our simulations and previously reported experimental studies. We show that the roughness theories capture the correct physics of deformation at the nanoscale. Our study provides a consistent explanation of the widely observed transition from a linear to sublinear dependence of the friction force on the applied load and we demonstrate that both regimes of friction are governed by the same physical phenomenon. Specifically, we show that friction is controlled by the mean number of atoms that interact chemically across the contact interface.
\end{abstract}

DOI: 10.1103/PhysRevB.81.035405

PACS number(s): 68.35.Af, 46.55.+d, 81.05.U-

\section{INTRODUCTION}

A continued miniaturization of devices has driven down their dimension down to the nanoscale. In particular microelectromechanical system (MEMS) and nanoelectromechanical system (NEMS) devices hold a tremendous potential in a variety of applications, such as resonance frequency switches, accelerometers, and pressure sensors. ${ }^{1}$ One important challenge in applications of MEMS devices is that at these small length scales surface forces (e.g., adhesion and friction) become dominant and can lead to a fast degradation of material's performance. ${ }^{1,2}$ Therefore, fundamental understanding of friction at the nanoscale is essential for the successful design of MEMS and NEMS devices.

Amontons' laws of macroscopic friction, ${ }^{3}$ which state that the friction force is proportional to the applied load and independent of the contact area, do not always apply to nanoscale or microscale contacts. ${ }^{4,5}$ Specifically, in microscale single asperity contacts $F_{\mathrm{f}}$ has been shown to be a sublinear function of load $L$ (Refs. 5-8) and this dependence is typically interpreted using continuum contact mechanics models. ${ }^{9}$ Continuum mechanics theories assume a simple geometry of a contact, i.e., a smooth spherical (or parabolic) asperity in contact with a smooth flat sample, where both contacting materials are linearly elastic solids. Another assumption often made when interpreting friction experiments is that the friction force $F_{\mathrm{f}}$ is proportional to the contact area. ${ }^{4,5}$ While continuum mechanics models have been quite successful in describing contact behavior at the microscale, a number of studies ${ }^{6,10-12}$ have suggested that continuum mechanics breaks down when the contact size reaches nanometer dimensions. For instance, both linear ${ }^{13-17}$ and sublinear ${ }^{4,6}$ dependences of the friction force on the applied load have been observed at the nanoscale. For macroscopic contacts, the linear dependence of the friction force on the load is conventionally explained by the theory of Bowden and Tabor, ${ }^{18,19}$ which is based on the assumption that the macroscopic contacts are rough and composed of a large number of small asperities. However, it is not well under- stood why $F_{\mathrm{f}}$ can depend linearly on $L$ for dry nanoscale or microscale single asperity contacts, which are typically regarded as smooth. Understanding of the fundamental mechanisms underlying the dependence of $F_{\mathrm{f}}$ on $L$ is essential for the interpretation of more complex frictional behavior, such as the transition from sublinear to linear $F_{\mathrm{f}}$ on $L$ dependence that can take place during sliding or when the chemistry of the interface is altered. ${ }^{12,15-17}$

Atomistic computer simulations, especially the moleculardynamics (MD) technique, have been widely used to study friction in nanoscale single asperity contacts. For instance, Luan and Robbins ${ }^{10,11}$ demonstrated that the atomic-scale surface roughness, which results from the discreteness of atoms on the surface, plays an important role at the nanoscale and that it leads to substantial deviations from predictions of continuum level contact mechanics. These authors also showed that the contact area of a nanoscale asperity is underestimated by continuum models, and that this deviation can be as large as a factor of 2. Friction and lateral stiffness may vary even by orders of magnitude. A detailed discussion of the breakdown of continuum mechanics can be found in Refs. 5 and 11. Although the importance of atomic-scale roughness has been demonstrated for nanoscale contacts, a satisfactory explanation is still lacking as to the origins of the different $F_{\mathrm{f}}$ vs $L$ dependences observed at these length scales. Both sublinear ${ }^{10,11,20}$ and linear ${ }^{6,21}$ dependences of $F_{\mathrm{f}}$ on $L$ has been reported in MD simulations and it has not been clear how these differences are related to the contact geometries and contact pressures, surface chemistry, and the interatomic potentials used in the simulations.

In a recent letter, ${ }^{22}$ we have demonstrated that because of the atomic roughness the real contact area, which is defined by the number of atoms interacting across the contact interface, can be much smaller than the area calculated based on the edge of the contact zone, which definition is commonly used in continuum mechanics. Our simulations have also shown that for dry, elastic, and wearless nanoscale contacts the friction force is always linear with the real contact area as long as the contact area is correctly defined at a given length scale. We have also shown that Amontons' laws are appli- 
cable for nanoscale contacts that have weak adhesion and high roughness. We discovered that an increase in interfacial adhesion in contact induces a transition from a linear to a sublinear behavior of the friction force on the applied load. Our results demonstrated that the discrepancies in the $F_{\mathrm{f}}-L$ behavior observed in previous MD simulations are due to the fact that these simulations explored different limits of contact behavior, i.e., some of the contacts were rougher than others.

In this paper, we revisit friction laws discovered for nanoscale dry contacts. More details on the simulations and on the comparison to experiments are provided. Additional results are reported for contacts with a varying radius of curvature. Simulations and analysis are also performed for contacts between two flat surfaces, which completes our earlier study that used spherical tips on a flat surface. We demonstrate that roughness theories, which were developed for microscale and macroscale contacts, can describe the mechanical behavior of nanoscale contacts in the regime of low loads. We quantify surface roughness of our atomic models of interfaces using a surface height profile. The estimated roughness parameters are compared to those reported in other MD studies to determine similarities and differences among these simulations. We show that often the differences in the $F_{\mathrm{f}}$ vs $L$ behavior observed in these MD simulations stem from exploring different limits of contact behavior. The origin of the transition between sublinear and linear dependences of $F_{\mathrm{f}}$ on $L$ is discussed in terms of the interplay between adhesion and roughness. Additionally, we have significantly extended the discussion of the existing theories of friction so that our findings can be put in the context of the literature.

\section{OVERVIEW: CURRENT UNDERSTANDING OF FRICTION LAWS}

Macroscopic laws of friction have been formulated by Amontons in $1699 .{ }^{3}$ According to these laws when two macroscopic bodies are in sliding contact, the resulting friction force $F_{\mathrm{f}}$ is linearly proportional to the applied load $L$, i.e., $F_{\mathrm{f}}=\bar{\mu} L$, where $\bar{\mu}$ is the macroscopic coefficient of friction. In addition, the coefficient of friction $\bar{\mu}$ is found to be independent of the applied load $L$ and of the macroscopic nominal contact area $A_{\text {macro }}$. Bowden and Tabor explained the physical basis of this behavior in metallic contacts. ${ }^{18,19,23,24}$ The authors noted that macroscopic surfaces are rough and comprised of a large number of small contacts (so-called asperities). The total contact area of these small asperities $\Sigma A_{\text {asp }}$ is orders of magnitude smaller than the macroscopic nominal contact area $A_{\text {macro }}$. The authors showed that the friction force $F_{\mathrm{f}}$ is proportional to this true contact area, i.e., $F_{\mathrm{f}}$ $=\bar{\tau} \sum A_{\text {asp }}$, where $\bar{\tau}$ is the effective shear strength of the interface. The true contact area $\Sigma A_{\text {asp }}$ has been demonstrated to depend linearly on load $L$. Bowden and Tabor interpreted this linear dependence of true contact area on load in terms of the plastic deformation of contacting asperities. ${ }^{24}$ Another quantitative model that explains the linear behavior has been proposed by Greenwood and Williamson $(\mathrm{GW}) .{ }^{25}$ In the GW model, a surface is approximated by a large number of asperities (protrusions), which deform elastically when two surfaces are in contact. The GW model assumes that asperities have a single uniform length scale, the deformation of each asperity is well described by Hertz model, and the elastic coupling between asperities is negligible. The height distribution of all asperities is assumed to be a Gaussian function. Although the GW model correctly predicts that linear relationship between the true contact area and the applied load, criticisms have been raised regarding some of the assumptions of this model, such as the predetermined single length scale of the asperities, a priori choice for the height distribution of asperities, and the lack of coupling between the elastic deformations of asperities. Recently efforts have been made to address the physical deficiencies of the GW model by incorporating into the model the distribution of asperity sizes, ${ }^{26}$ plastic deformation and wear of the asperities, ${ }^{27}$ adhesion across the contact interface, ${ }^{28,29}$ and elastic coupling between asperities. ${ }^{30}$ A self-assessment of the GW model can be found in Ref. 31 .

A different approach to describing deformation in contacts with random roughness has been proposed by Persson and co-workers. ${ }^{32-35}$ In this model, pressure distribution in contact and the contact area are determined by the autocorrelation function of the surface profile. One major physical advantage of Persson's model is that it treats multiple length scales of roughness simultaneously and no specific length scale of roughness is excluded. A critical overview of Persson's model can be found in Ref. 36. Persson's model predicts that the pressure distribution is described by a doubleGaussian function, i.e.,

$$
P(p)=\frac{1}{\sigma \sqrt{2 \pi}}\left\{\exp \left[-\frac{\left(p-p_{0}\right)^{2}}{2 \sigma^{2}}\right]-\exp \left[-\frac{\left(p+p_{0}\right)^{2}}{2 \sigma^{2}}\right]\right\},
$$

where $p_{0}$ and $\sigma$ are the peak and width of the Gaussian distributions, respectively. In the limit of low loads, Eq. (2.1) becomes: ${ }^{37}$

$$
P(p)=\frac{\pi}{2} \cdot \frac{p}{\langle p\rangle^{2}} \cdot \exp \left[-\frac{\pi}{4}\left(\frac{p}{\langle p\rangle}\right)^{2}\right],
$$

where $\langle p\rangle$ is the average pressure calculated over the real contact area. While Persson's distribution is normalized by the apparent contact area, the distribution shown in Eq. (2.2) is normalized by the real contact area. In the low load regime, the pressure distribution is independent of the applied load. ${ }^{37}$

Macroscopic friction depends not only on roughness of the surfaces in contact, but also on other interfacial properties, such as atomic structure of surfaces, chemistry of the interface, elastic and plastic properties of the contacting materials, the environment in which measurements are performed, the setup of the experimental instruments, and in many cases also the sliding history. Because of the complex nature of the energy dissipation mechanisms underlying friction, fundamental understanding of these mechanisms requires studies at well-defined interfaces. Such studies have been enabled by the developments in experimental techniques, such as the scanning force microscope (SFM). ${ }^{38}$ Thanks to the state of the art SFM techniques, it is now 
TABLE I. Friction laws for macroscopic, microscale single asperity, and nanoscale contacts (proposed here). $A_{\text {macro }}$ is the macroscopic contact area, $A_{\text {asp }}$ is the contact area of a single asperity (for SFM tip radii from tens of nanometers to micrometers in size), and $A_{\text {real }}$ is the real contact area defined as the number of atoms $\left\langle N_{\text {at }}\right\rangle$ in contact multiplied by the surface area $A_{\text {at }}$ of an atom. $A_{0}$ and $L_{0}$ are small offsets in the linear relationships of $F_{\mathrm{f}}$ on $L$ and $F_{\mathrm{f}}$ on $A_{\text {real }}$, respectively.

\begin{tabular}{|c|c|c|c|c|c|}
\hline \multirow[b]{2}{*}{ Friction laws } & \multirow{2}{*}{$\begin{array}{c}\begin{array}{c}\text { Macroscale } \\
\text { theories }\end{array} \\
\text { Amontons' laws } \\
\text { (Bowden and Tabor) }\end{array}$} & \multicolumn{2}{|r|}{$\begin{array}{l}\text { Single asperity } \\
\text { theories }\end{array}$} & \multicolumn{2}{|c|}{$\begin{array}{l}\text { Our model: } \\
\text { Multiasperity picture } \\
\text { of nanoscale contact }\end{array}$} \\
\hline & & $\begin{array}{l}\text { Nonadhesive } \\
\text { (Hertz model) }\end{array}$ & $\begin{array}{c}\text { Adhesive } \\
\text { (e.g., Maugis-Dugdale model) }\end{array}$ & Nonadhesive & Adhesive \\
\hline$F_{\mathrm{f}}$ vs Area & $\begin{array}{l}\text { Independent of } A_{\text {macro }} \\
\qquad F_{\mathrm{f}}=\bar{\tau} \Sigma A_{\text {asp }}\end{array}$ & $F_{\mathrm{f}}=\tau A_{\mathrm{asp}}$ & $F_{\mathrm{f}}=\tau A_{\mathrm{asp}}$ & $\begin{array}{c}F_{\mathrm{f}} \propto N_{\mathrm{at}} \\
F_{f}=\tau\left(A_{\text {real }}-A_{0}\right)\end{array}$ & $\begin{array}{c}\quad F_{\mathrm{f}} \propto N_{\text {at }} \\
F_{f}=\tau\left(A_{\text {real }}-A_{0}\right)\end{array}$ \\
\hline Area vs $L$ & $\Sigma A_{\text {asp }} \propto L$ & $A_{\text {asp }} \propto L^{2 / 3}$ & Sublinear & $A_{\text {real }} \propto L$ & Sublinear \\
\hline$F_{\mathrm{f}}$ vs $L$ & $F_{\mathrm{f}}=\bar{\mu} L$ & $F_{\mathrm{f}} \propto L^{2 / 3}$ & Sublinear & $F_{f}=\mu\left(L-L_{0}\right)$ & Sublinear \\
\hline
\end{tabular}

possible to measure friction at a single asperity level, ${ }^{6,10,11,21,22}$ where the contact consists of a flat sample and a relatively smooth parabolic tip with the radius of curvature between tens of nanometers to micrometers. At the single asperity level, the friction force $F_{\mathrm{f}}$ is usually regarded as being proportional to the contact area $A_{\text {asp }}$, i.e., $F_{\mathrm{f}}=\tau A_{\text {asp }}$. The dependence of the contact area $A_{\text {asp }}$ on the applied normal load $L$ is described by continuum contact theories. ${ }^{9}$ In these models, the two contacting bodies are assumed to be homogeneous, isotropic, and linear elastic continuum solids with perfectly smooth surfaces. The field of continuum contact mechanics has been pioneered by Hertz ${ }^{39}$ who in 1881 derived the first analytical model to describe contacts, where the interfacial interaction between the sphere and the flat surface is an infinitely sharp hard-wall repulsion. The Hertz model predict the contact area of the asperity as

$$
A_{\text {asp }}=\pi\left(3 R / 4 E^{*}\right)^{2 / 3} L^{2 / 3},
$$

where $R$ is the radius curvature of the tip;

$$
E^{*}=\left[\left(1-v_{1}^{2}\right) / E_{1}+\left(1-v_{2}^{2}\right) / E_{2}\right]^{-1}
$$

is the effective modulus of the contact; $E_{1}$ and $E_{2}$ are the tip and the sample Young's moduli; and $v_{1}$ and $v_{2}$ are Poisson's ratios of the tip and the sample, respectively. There is no interfacial adhesion in the Hertz model. To account for the interfacial adhesion, adhesive continuum contact models have been subsequently developed. In particular, Johnson, Kendall, and Roberts (JKR) (Ref. 40) proposed a model, where the interfacial adhesion is described by infinitely short-range attractive forces, i.e., the adhesion is present only for those parts of surfaces that are in an intimate contact and is zero outside of the contact area. In another model developed by Derjaguin, Muller, and Toporov (DMT) ${ }^{41}$ interfacial adhesion is represented by a long-range attractive interaction. The DMT model can be represented analytically as an offset load added to the Hertz model. ${ }^{42}$ The JKR and DMT models predict different functional dependences between $A_{\text {asp }}$ and $L$. It is now understood that the JKR and DMT models describe deformation at two opposite ends of the contact behavior. $^{42-44}$ The JKR model is more appropriate for compliant materials, large sphere radii, and a strong short-range adhesion. On the other hand, DMT model de- scribes well a situation where a stiff material is in contact with a tip that has a small curvature radius and where the interfacial adhesion is weak and long range. A transition between the JKR to the DMT limits has been described by a transition parameter $\mu_{\mathrm{T}}$ proposed by Tabor. ${ }^{43}$ This so-called Tabor's parameter is defined as

$$
\mu_{\mathrm{T}}=\left(\frac{R \gamma^{2}}{E^{* 2} z_{0}^{3}}\right)^{1 / 3}
$$

where $z_{0}$ is surface equilibrium separation and $\gamma$ is defined as adhesion energy per unit area. The total adhesion energy is defined as the work required to separate two surfaces in contact and to move them infinitely apart. In SFM experiments, the value of $\gamma$ is typically obtained from the pull-off force measurement. By estimating the value of $\mu_{\mathrm{T}}$, it can be determined whether the contact behavior should be described by the JKR or DMT model. For $\mu_{\mathrm{T}}>5$, the JKR model gives an accurate prediction of the contact behavior and DMT model is a better approximation in the limit $\mu_{\mathrm{T}}<0.1$. Intermediate cases are quantitatively described by the so-called Maugis-Dugdale ${ }^{42}$ model, which includes JKR and DMT as the limiting cases. Another intermediate model has been proposed by Schwarz where both short- and long-range adhesion interactions are considered simultaneously inside and outside the contact zone. ${ }^{45}$ Irrespectively of the details, all single asperity continuum models predict a sublinear dependence of $A_{\text {asp }}$ on $L$ and consequently of $F_{\mathrm{f}}$ on $L$. This prediction is in contrast to the behavior of rough macroscopic contacts where $F_{\mathrm{f}}$ is a linear function of the applied load $L$. A summary of the friction laws for both macroscopic and single asperity contacts is provided in Table I.

The single asperity continuum contact models have been demonstrated to successfully describe friction and contact behavior of microscale contacts. ${ }^{12,46}$ For example, friction and contact area directly measured by surface force apparatus (SFA) frequently follow predictions of the Hertz model. ${ }^{46-48}$ However, when the size of the contact is reduced down to the nanometer regime a number of studies reported deviations from the continuum models. ${ }^{6,10-12}$ The observed deviations have been attributed to the breakdown of some of the assumptions of continuum models at these small length 
scales. For instance, surfaces can be no longer regarded as perfectly smooth because in nanoscale contacts atomic-scale roughness can have a significant effect on the contact area and pressure distribution. ${ }^{10,11,22}$ Additionally, the interfacial interaction has a finite compliance, in contrast to the hardwall repulsion assumed in continuum models. ${ }^{9,49}$ Continuum models also assume that the lateral stiffness of the contact is entirely due to the elastic deformation of the two contacting bodies. In contrast, for real interfaces the lateral stiffness arises also from changes in the interfacial bonding that take place during sliding and from the local relaxation of interfacial atoms to their minimum energy position. ${ }^{50,51}$

A number of studies reported that physical quantities predicted by continuum models deviate from their true values. ${ }^{6,10,11}$ For instance, Luan and Robbins showed that the contact area measured directly in their simulation is larger than that predicted by the Hertz model..$^{10,11}$ The assumption that the friction force is proportional to the contact area has also been questioned. ${ }^{11,12,20,21,52}$ A number of experimental studies ${ }^{13-17}$ have shown that in nanoscale single asperity contacts $F_{\mathrm{f}}$ does not scale with contact area but it is linearly proportional to $L$ for both dry and lubricated surfaces. The contact area of nanoscale contacts is not only difficult to measure, but also difficult to define because at this length scale the edge of the contact zone is not uniquely defined. ${ }^{11,53}$ The discussion of different definitions of contact area can be found in Sec. IV A. Various solutions have been put forth to explain the fact that the friction force does not scale linearly with the contact area. Gao and co-workers ${ }^{12}$ proposed that contact area is not a useful quantity to describe friction at the nanoscale. Wenning and Müser ${ }^{20}$ suggested that the friction force is not determined by contact area itself, i.e., the shear strength is not a constant, but instead it varies with the load.

Because of the above uncertainties associated with the dependence of the friction force on the applied load and the contact area, both continuum contact models as well as the original Amontons equation are currently used to describe the frictional behavior of nanoscale contacts. The regime where the friction force $F_{\mathrm{f}}$ is proportional to the applied load $L$ has been referred to as load-controlled friction. ${ }^{8,12}$ Currently, there is lack of a theory that would allow the interpretation of the linear dependence of $F_{\mathrm{f}^{-}} L$ for single asperity contacts. The conventional explanation of this relation for macroscopic contacts, e.g., the Bowden and Tabor theory, is based on the assumption that the rough contact composes multiple asperities. ${ }^{12}$ This assumption cannot be applied when the entire contact consists of a single asperity. The regime where $F_{\mathrm{f}}$ is a sublinear function of load is referred to as adhesion-controlled friction. ${ }^{8,12}$ In this regime, the friction force $F_{\mathrm{f}}$ is proportional to the contact area and the relationship between the contact area and load follows the prediction of continuum mechanics. Furthermore, a transition from load-controlled to adhesion-controlled friction (also known as linear to sublinear transition of the $F_{\mathrm{f}}-L$ dependence) has been widely observed in experiments for dry ${ }^{13,16}$ and lubricated $^{15,17}$ contacts. To account for the two different regimes of friction and the transition between them, Israelachvili and Berman ${ }^{8}$ proposed a phenomenological model that describes the friction force as a sum of two separate contributions, i.e.,

$$
F_{\mathrm{f}}=\mu L+\tau A_{\mathrm{asp}},
$$

where the first term dominates in the load-controlled regime of friction and the second term dominates in the adhesioncontrolled regime. A theory is needed to account for the physical origin of these two different friction regimes and for the transition between these regimes.

It is challenging to fully address the above questions by experimental techniques alone. Not only contact areas cannot be measured directly in SFM experiments, but also much of the reported SFM data on friction are not accompanied by a detailed surface characterization, i.e., information about surface atomic structure and composition is often not provided. Frictional response of materials is by nature highly sensitive to specific experimental conditions. Computer simulations, on the other hand, can be very helpful in addressing some of the ambiguities encountered in experimental studies. Combining simulations with experiments can be a particularly powerful approach to isolating and identifying specific contributions to friction. Among many simulation methods, the MD technique is likely the one that has been most frequently used to model friction behavior at the atomic level. Major advantages of MD simulations include the ability to track the dynamics of every atom in the system, a precise control over the simulation conditions, and the ability to directly measure a number of key physical quantities, such as the contact area, the stress distribution, and forces experienced by individual atoms. ${ }^{5,54}$ Thanks to the developments of MD parallelization techniques, the system sizes accessible to the state of the art MD simulations are now on the micrometer length scale and they are comparable to the length scales of advanced SFM experiments. MD simulations have also some limitations. One challenge is the limited time scale accessible to the simulations. Standard MD simulations are carried out with sliding velocities on the order of $0.1-100 \mathrm{~m} / \mathrm{s}$, which is five to nine orders of magnitude higher than nominal sliding velocities in SFM experiments. Time-accelerated MD techniques are being developed to address this challenge. ${ }^{55,56} \mathrm{~A}$ discussion of these techniques and their applicability to nanotribology can be found in Ref. 5. An interatomic potential (also known as a force field) is another critical issue for the reliability of MD simulations. Although relatively simple potentials, such as the harmonic spring interaction or LennardJones potential, have been used in friction studies to determine the dependence of friction on the interface geometry, ${ }^{10,11,20,57}$ advanced many-body interatomic potentials are needed if one wants to investigate the effects of surface chemistry and structure on mechanical response. ${ }^{21}$ It is also important to set up the atomistic model so that it provides a realistic representation of experimental conditions, e.g., to choose boundary conditions on the system that have a limited effect on the deformation in the contact itself. An approximation commonly used in MD simulations is to treat one of the two contacting bodies as infinitely stiff, i.e., the coordinates of atoms are fixed in space. While such an approach is reasonable in many cases (e.g., when the focus of the study is wear of one of the contacting materials), the effect of the frozen atoms on frictional resistance may not be neglected when contact pressures and friction forces are 
compared between simulations and experiments.

Despite the tremendous insights brought by MD simulations to friction studies, seemingly contradictory results regarding friction laws, i.e., how the friction force depends on the applied load, are still being reported. For instance, for amorphous or incommensurate interfaces both Luan and Robbins ${ }^{10,11}$ and Wenning and Müser ${ }^{20}$ reported a friction force $F_{\mathrm{f}}$ that scales with the applied load as $L^{2 / 3}$, whereas Gao et al. ${ }^{6}$ reported that $F_{\mathrm{f}}$ depends linearly on $L$. There may be multiple origins of the observed discrepancies, e.g., they could be related to the differences in atomic roughness of the interfaces, size of the SFM tips, possibly different scaling laws for static and kinetic friction, and the magnitude of pressure reached in the contacts. The origins of the observed differences in the dependence of $F_{\mathrm{f}}$ on $L$ are yet to be determined for both simulations and experiments.

\section{SIMULATION METHODS}

Molecular-dynamics simulations are carried out using our in-house code. To correctly describe interactions between atoms, we use the second generation reactive empirical bondorder (REBO) potential. ${ }^{58}$ The REBO potential has been successfully used in MD simulations of tribochemistry. ${ }^{59,60}$ This potential accurately describes atomic interactions of hydrocarbon systems, including the atomic structures of both diamond and diamond-like carbon (DLC), elastic properties of these solids, surface energies of diamond, and breaking and forming of covalent bonds. Therefore, both mechanical deformations and chemical reactions of the hydrocarbon systems are correctly handled by REBO, which makes it an excellent potential for studies of friction. The range of the REBO potential extends as far as the covalent bonding and REBO does not account for dispersive forces. This version of the REBO potential is used in our simulations of nonadhesive contacts.

To properly describe the interfacial interactions for adhesive contacts, we integrated van der Waals (vdW) interactions with the REBO potential. The original parameters and properties of the REBO potential are unaltered. A smooth transition between REBO and vdW is realized using an analytical switching function in the regime where the two potentials overlap. The total vdW energy can be written as

$$
E_{\mathrm{vdW}}\left(r_{i j}\right)=-\frac{1}{2} \sum_{i} \sum_{j \neq i} C_{i j} \cdot 4 \varepsilon_{i j}\left(\frac{\sigma_{i j}}{r_{i j}}\right)^{6},
$$

where $r_{i j}$ is the interatomic distance, $C_{i j}$ is the aforementioned switching function, and $\sigma_{i j}$ and $\varepsilon_{i j}$ are vdW parameters. The vdW interaction is included only for those atoms $i$ and $j$ that interact across the contact interface. The values of $\sigma_{i j}$ are chosen to be 3.4 and $2.65 \AA$, and the values of $\varepsilon_{i j}$ are chosen to be 1.42 and $0.75 \mathrm{meV}$, for $\mathrm{C}-\mathrm{C}$ interactions and $\mathrm{H}-\mathrm{H}$ interactions, respectively. The above values are the same as in the adaptive intermolecular REBO potential. ${ }^{61}$ The only exception is that we decreased the value of $\varepsilon_{i j}$ by $50 \%$ in order to correctly reproduce the experimental value of a pull-off force measured with a DLC tip on diamond. ${ }^{6}$

In our MD simulations of SFM experiments we use a flat diamond sample and a DLC spherical tip. Two different samples are used: one with dimensions of $49.9 \times 48.0$ $\times 12.2 \mathrm{~nm}^{3}$ and the other with dimensions of $39.4 \times 37.9$ $\times 12.2 \mathrm{~nm}^{3}$. The former sample is used in conjunction with the tip of radius of curvature $R$ equal to $30 \mathrm{~nm}$. The latter sample is used for tip sizes $R$ of 5,10 , and $20 \mathrm{~nm}$. The diamond sample is terminated with hydrogen atoms and the sliding is performed on the (111) surface. The sample is equilibrated at $300 \mathrm{~K}$ prior to the loading and sliding simulation. All SFM tips have the same height of $10 \mathrm{~nm}$. The dimensions of our systems are comparable to those encountered in experiments. ${ }^{6}$

The SFM tips are prepared by cutting the desired shape out of a DLC bulk. The tips are first relaxed at $0 \mathrm{~K}$ using molecular static simulations to reduce surface energy. Subsequently $\mathrm{H}$ atoms are placed on the tip surface to terminate unsaturated $\mathrm{C}$ bonds. After $\mathrm{H}$ passivation, each tip is gradually heated up to and equilibrated at $300 \mathrm{~K}$ using MD. In addition, the bottom four atomic layers in each sample are held rigid during the simulations of normal loading and lateral sliding. To dissipate excessive thermal energy generated by loading and sliding, Langevin thermostat with an inverse time constant of $0.01 \mathrm{~s}^{-1}$ is applied to a $1 \mathrm{~nm}$ thick layer of atoms, which is adjacent to the rigid layers. Periodic boundary conditions are applied in both lateral directions. Similar combination of rigid and thermostat layers is applied to the tip. In our earlier study (see supplementary information in Ref. 22) we used finite element analysis to test the effect of the finite system size and of the specific tip geometries and we have shown that these effects are negligible. That means that any deviations from continuum contact mechanics observed in our MD simulations can be attributed to the discreteness of atoms. In or simulations all atoms (except those in the rigid layers) of both the tip and the sample are allowed to deform and relax dynamically at $300 \mathrm{~K}$. In addition, no special treatment is applied to the interface atoms, and therefore all chemical interactions are allowed to occur as described by the REBO potential.

First, we perform simulations of normal loading, i.e., nanoindentation with SFM tips, to study the contact mechanics, i.e., the relationship between the contact area and the applied load. Simulations of normal loading are performed by altering between loading and holding phases. In the loading phase, the tip is displaced toward the sample in the increments of 0.25 and $0.1 \AA$ for the nonadhesive and adhesive cases, respectively, and each loading step takes 2.5 ps. The holding phases last 10 and 6 ps, respectively, which is long enough for transient forces to decay. The average indentation speeds are 2.5 and $1.7 \mathrm{~m} / \mathrm{s}$ for the nonadhesive and adhesive simulations, respectively.

For each normal displacement (and therefore each normal load), friction simulations are performed by laterally sliding the tip over the surface in the $(2 \overline{1} \overline{1})$ direction of the diamond (111) surface. The sliding velocity is $20 \mathrm{~m} / \mathrm{s}$. While this velocity is comparable to the operating conditions in MEMS, it is a few orders of magnitude larger than the typical velocity in SFM experiments. ${ }^{6}$ Bridging the existing gap in sliding velocities between MD and SFM still remains one of the outstanding challenges in the field. One validation of our MD simulations come from the fact that the calculated shear 
strengths and contact pressures quantitatively agree with the corresponding values measured in experiments as discussed in Sec. IV. Additional validation and discussion of issues associated with the high sliding velocity in our MD simulations can be found in Sec. V. During sliding simulations we record all three components of the net force that acts on the tip. These net forces are calculated directly by summing up forces on all the tip atoms. The oscillations of the friction force over time are consistent with the periodicity of the surface lattice of the diamond sample. We calculate normal and friction forces by averaging them over each lattice period of the surface lattice. The reported values are averaged over three to five lattice periods, and error bars in the figures correspond to standard deviation from these averages. The standard deviation of the load is approximately $1 \mathrm{nN}$, which is smaller than the symbol size in the respective figures. The above scheme allows us to determine and quantify relationships between friction, contact area, and load.

\section{RESULTS}

\section{A. Definition of contact area}

In the Hertz model, where two smooth and continuous bodies are in contact, the definition of contact area is straightforward. A region of a surface is defined as being in contact if it experiences a positive (repulsive) normal stress. Contact area is defined as the entire area enclosed by the edge of the contact zone, which is reasonable because the surfaces are assumed to be smooth. The edge of the contact zone is defined as the point where the normal contact stress or pressure decreases to zero. The edge of the contact zone is also well defined in the JKR model, where the adhesive interaction has an infinitely short range. However, the definition of a contact edge becomes ambiguous when the interfacial adhesive interaction has a finite range, such as in the Maugis-Dugdale model. The finite range of the adhesive interaction leads to formation of a region of a tensile (negative) stress outside of the intimate contact zone. ${ }^{11,53}$ Different choices can be made as to how far the contact zone extends into the tensile region. For example, both the innermost edge (the point where the normal pressure changes from positive to negative) and the outermost edge (the point where the normal pressure decreases to zero) of the tensile stress region have been used to define the contact area. ${ }^{42,44,53}$ The edge of contact has been also defined as the point where the negative pressure reaches its peak value. ${ }^{11,62}$ The different definitions of contact area has been systematically studied by Luan and Robbins ${ }^{11}$ using MD simulations. It was shown that a specific definition of the contact edge does not affect the dependence of the friction force on the contact area, except for the offset from the origin on the $F_{\mathrm{f}}$ vs $A_{\text {asp }}$ plot.

The definition of contact area becomes even less straightforward for interfaces where discreteness of atoms is the dominant structural feature. We define a surface atom as being in contact if this atom is within the range of chemical interaction of any atom of the counter surface. This chemical interaction is either a steric repulsion or a chemical bonding, both of which are uniquely defined in the REBO potential. These interactions represent an overlap of electronic densi- ties and therefore this definition of contact is universal among all the atomic systems. Although a specific cutoff range is selected as the range of interactions in the REBO potential in our simulations, our definition of contact area is not specifically constrained to a given potential. Additionally, for nonadhesive contacts, where interactions between surface $\mathrm{H}$ atoms are purely repulsive, our criterion for an atom to be in contact is consistent with the continuum mechanics description of contact defined as part of the surface that experiences a positive normal load. A snapshot of the interface showing atoms in contact at a normal load $L=71 \mathrm{nN}$ is shown in Fig. 1(a).

Our simulations are carried out at $300 \mathrm{~K}$. Due to the thermal fluctuations, surface atoms may come in and out of contact during the simulation. The instantaneous number of atoms in contact $N_{\text {at }}$ is calculated at each MD time step (equal to $0.5 \mathrm{fs}$ ) after the modeled system has reached the steady state. While the instantaneous value of $N_{\text {at }}$ fluctuates [diamond symbols in Figs. 1(b) and 1(c)], the mean value $\left\langle N_{\text {at }}\right\rangle$ of this quantity remains constant over time (open symbols in the above figures). It is the mean value $\left\langle N_{\text {at }}\right\rangle$ that we use in our definition of contact area. Estimating $\left\langle N_{\text {at }}\right\rangle$ is analogous to calculating an average number of bonds formed across the contact interface, except that some of the atoms that contribute to $N_{\text {at }}$ have purely repulsive interactions with the counterface and therefore do not form chemical bonds. It is important to note that the definition of $\left\langle N_{\text {at }}\right\rangle$ does not assume that the same atoms are in contact at all times. This definition also is different from counting the total number of distinct atoms that ever came into contact or that came into contact over a specific period of time. Cheng et al. ${ }^{63}$ recently showed that the number of distinct atoms that come into contact within a certain time interval increases with the length of this interval due to thermal fluctuations. In contrast, our definition of $\left\langle N_{\mathrm{at}}\right\rangle$ yields a constant value regardless of the time interval over which we calculate the average. It might be useful to draw an analogy between our calculations of atoms in contact and the pressure of gas. In the kinetic theory of gasses, pressure is calculated as the number of collisions of gas particles with a wall of a container per unit time. Pressure on the surface can be also calculated as the number of collisions per unit time, which is equivalent to the number of atomic interactions across contact interface or the mean number of atoms in contact $\left\langle N_{\text {at }}\right\rangle$. In equilibrium the pressure and therefore also the value of $\left\langle N_{\text {at }}\right\rangle$ are constant. The equivalence between the number of atoms and the number of collisions will hold only if each atom makes no more than one collision within the time interval of the calculations. While the instantaneous number of atoms in contact (averaged over time) satisfies this condition, this condition will not be true if one counts each atom that ever came into contact with the counter surface within a time period that is large compared to the vibrational period of a surface atom. The next question is how to convert a discrete number $\left\langle N_{\text {at }}\right\rangle$ of atoms in contact into a quantity that has units of the area. Here, we define the real contact area $A_{\text {real }}$ as

$$
A_{\text {real }}=\left\langle N_{\text {at }}\right\rangle A_{\text {at }},
$$

where $A_{\text {at }}$ is the average surface area per atom [hexagons in Fig. 1(a)]. The value of $A_{\text {at }}$ is calculated by dividing the total 


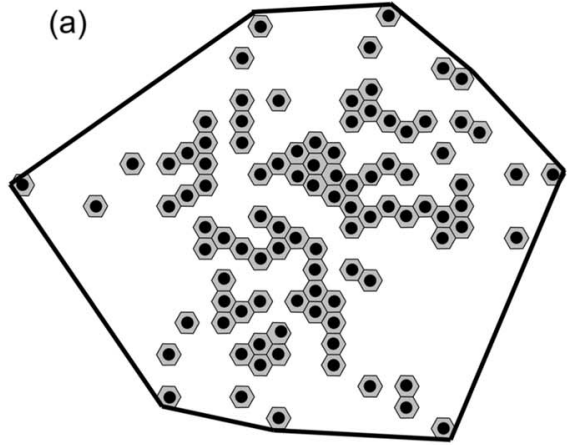

Average surface area per surface atom - Edge of contact zone

- Atoms on the sample's surface in contact with the tip
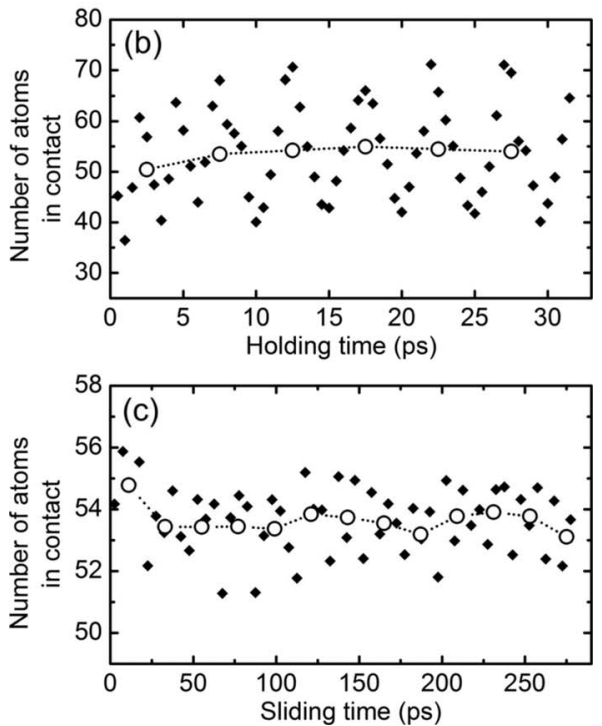

FIG. 1. (a) Definitions of the contact area. A snapshot of the atoms in contact for $30 \mathrm{~nm}$ tip at a normal load of $71 \mathrm{nN}$. Full circles represent atoms of the sample that are in contact with the tip, i.e., they are within the range of chemical interaction from the tip atoms. Each gray hexagon is the average surface area of a surface atom. Real contact area $A_{\text {real }}$ is defined as the total area of these hexagons. In analogy to continuum contact models, the asperity contact area $A_{\text {asp }}$ is defined by the convex hull enclosing all contact atoms. The edge of the contact zone is marked by the solid line. (b),(c) Number of atoms in contact $N_{\text {at }}$ plotted as a function of time (b) during holding of the tip in place and (c) during sliding of the tip at a finite normal load. Filled diamond symbols correspond to $N_{\text {at }}$ averaged over (b) 0.5 and (c) 5 ps, and open circles correspond to an average over (b) 5 and (c) 22 ps. While the quantity $N_{\text {at }}$ fluctuates due to thermal vibrations, its mean value $\left\langle N_{\mathrm{at}}\right\rangle$ is constant over time.

surface area of a sample by the total number of surface atoms (in our case $\mathrm{H}$ atoms). A similar method to quantify $A_{\text {at }}$ and $A_{\text {real }}$ has been used in a number other studies. ${ }^{6,64,65}$

To test the predictions of continuum mechanics models in nanoscale contacts, we also define a contact area using the concept of the edge of the contact zone. We refer to this area as the asperity contact area $A_{\text {asp }}$, in contrast to the real contact area $A_{\text {real }}$. Here, the edge is defined as a two-dimensional convex hull of the atoms in contact and $A_{\text {asp }}$ is defined as the

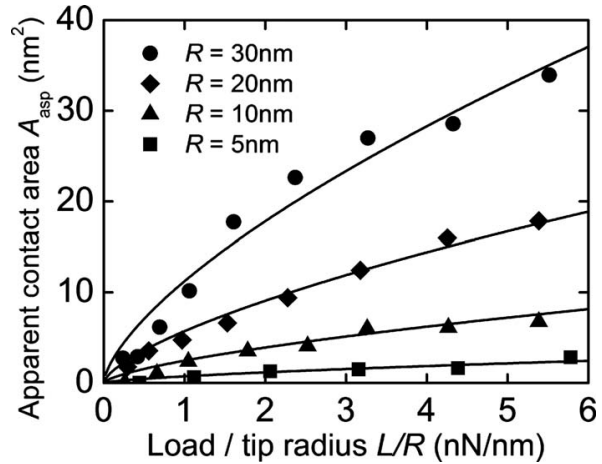

FIG. 2. The dependence of the asperity contact area $A_{\text {asp }}$ on the applied load $L$ for nonadhesive contacts. Symbols correspond to DLC tips with different radii of curvature $R=30,20,10$, and $5 \mathrm{~nm}$. Solid lines represent fits to Hertz model.

area enclosed by this convex hull. The definition of $A_{\text {asp }}$ is sensitive to the specific choice of which atoms are counted as being in contact, and not just how many atoms are in contact on the average. Since the surface atoms are vibrating, some of the atoms are in contact only for a fraction of the simulation time. We select atoms in contact to be those that spend $30 \%$ or more time in contact. This value was chosen so that the number of atoms that meets this criterion is equal to $\left\langle N_{\text {at }}\right\rangle$, which is the time average of the instantaneous number of atoms in contact as defined in previous paragraphs. Our simulations show [Fig. 1(a)] that not every atom within the edge of the contact zone is actually in contact, which means that the contact area is rough. In the subsequent sections we discuss the effect of this surface roughness on friction laws and specifically we show how the friction force and applied load scale with these two definitions of contact area.

\section{B. Area-load dependence for nonadhesive contacts}

We first test the applicability of the Hertz model to nanoscale nonadhesive contacts by performing MD simulations in the absence of vdW interactions. Although the chemical reactions are still allowed at the interface (i.e., in principle atoms can form chemical bonds), adhesion is negligible during the entire simulation due to the fact that both the tip and the sample are perfectly passivated with $\mathrm{H}$ atoms and the applied load is low. Thus, if the continuum mechanics is valid at this length scale, the contact behavior is expected to be consistent with the Hertz model.

In Fig. 2 we show how the asperity contact area $A_{\text {asp }}$, which is defined by the edge of contact zone, depends on the applied load $L$ in MD simulations of normal loading. The solid lines represent fits of the MD data to the Hertz model. For all tip sizes considered $(R=5,10,20$, and $30 \mathrm{~nm}), \mathrm{MD}$ data show a qualitative agreement with the Hertz model, i.e., $A_{\text {asp }} \propto L^{2 / 3}$. The small deviation from Hertzian dependence may be a result of an error in the estimate of $A_{\text {asp. }}$. This error is induced by the random height variance of the tip atoms and the fact that $A_{\text {asp }}$ is sensitive to the number and exact positions of atoms at the edge of a contact zone [see Sec. IV A and Fig. 1(a)]. 

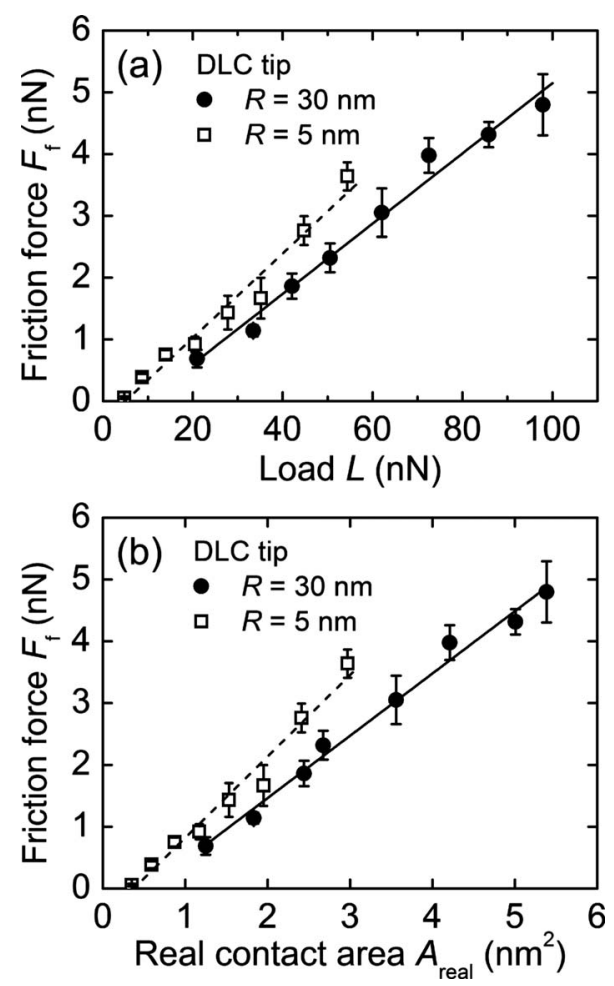

FIG. 3. Friction in nonadhesive contacts. The dependence of friction force $F_{\mathrm{f}}(\mathrm{a})$ on the applied load $L$ and (b) on the real contact area $A_{\text {real }}$ for DLC tips with radii $R=5 \mathrm{~nm}$ (squares) and $30 \mathrm{~nm}$ (circles). The solid and dashed lines represent linear fits to MD data.

A quantitative comparison of MD data with predictions of Hertz model is performed by extracting the effective modulus of contact $E^{*}$ from the fits to the simulation data [Eq. (2.3)]. The values obtained from the fits are 118, 114, 131, and $137 \mathrm{GPa}$ for tip radii of $30,20,10$, and $5 \mathrm{~nm}$, respectively. The differences among these values are likely due to the uncertainties in our calculations of $A_{\text {asp }}$. The theoretical value of $E^{*}$ equals $303 \mathrm{GPa}$, which is calculated from Eq. (2.4) based on the elastic modulus of the DLC tip $\left(E_{1}\right.$ $=348 \mathrm{GPa}$ and $\left.v_{1}=0.33\right)$ and the diamond sample $\left(E_{2}\right.$ $=1330 \mathrm{GPa}$ and $\left.v_{2}=0.11\right)$. The effect of the anisotropy of the diamond sample on $E^{*}$ was estimated based on the theory outlined in Refs. 66 and 67 and this effect was found to be negligible (as small as 1\%). This analysis shows that while the Hertz model qualitatively describes the dependence of the asperity contact area on the applied load even in nanoscale contacts, there are quantitative discrepancies. Specifically, the difference between the values of $E^{*}$ obtained from fitting and the ones calculated from the definition of $E^{*}$ can be as large as $54-62 \%$. Significant quantitative discrepancies between the simulation results and the Hertz model has been also reported by Luan and Robbins. ${ }^{10,11}$

\section{Friction of nonadhesive contacts}

We perform simulations of sliding to determine the dependence of the friction force on the contact area and load for nonadhesive contacts. As shown in Fig. 3(a), the measured friction force $F_{\mathrm{f}}$ is linear with the applied load $L$, i.e.,
$F_{f}=\mu\left(L-L_{0}\right)$, where $L_{0}$ is a small offset. This behavior is consistent with Amontons' laws rather than with the Hertz model, which predicts that $F_{\mathrm{f}} \propto L^{2 / 3}$. The friction coefficient $\mu$ calculated from our MD simulations for diamond equals 0.05 . This value of $\mu$ is in agreement with the experimentally measured macroscopic coefficient of friction of $0.05 .^{18,68,69}$ The agreement of friction coefficients between nanoscale and macroscale measurements has been previously observed in experiments carried out on a number of other materials. ${ }^{14}$ It is worth noting that in Figs. 3(a) and 3(b), the friction force $F_{\mathrm{f}}$ vanishes at finite values of $L$ and $A_{\text {real }}$, i.e., the linear relationships of $F_{\mathrm{f}}$ on $L$ and $F_{\mathrm{f}}$ on $A_{\text {real }}$ have small offsets $L_{0}$ and $A_{0}$, respectively. A similar offset at such low loads has also been observed experimentally by Socoliuc et al. ${ }^{50}$ and in MD simulations by Cheng et al. ${ }^{63}$

The results of our MD simulations have shown that $F_{\mathrm{f}}$ $\propto L$ and $A_{\text {asp }} \propto L^{2 / 3}$ (see Sec. II). Therefore, $F_{\mathrm{f}}$ is not a linear function of the contact area $A_{\text {asp }}$ and the widely used assumption that $F_{\mathrm{f}}=\tau A_{\text {asp }}$ breaks down. Our conclusion agrees with those of other authors ${ }^{12,20}$ that the asperity contact area does not control friction or rough nanoscale contacts. The lack of linear scaling of $F_{\mathrm{f}}$ with $A_{\text {asp }}$ has also been observed in some SFA experiments at the micrometer scale. ${ }^{47,48}$ We propose that the friction force is not controlled by $A_{\text {asp }}$ because the nanoscale contact is rough. The roughness of contact area in the nanoscale contact is evidenced by the fact that the real contact area $A_{\text {real }}$ is significantly smaller than the asperity contact area $A_{\text {asp }}$ (see Sec. IV A). Interestingly, our simulation data reveal that the friction force $F_{\mathrm{f}}$ scales with the real contact area $A_{\text {real }}$, i.e., $F_{f}=\tau\left(A_{\text {real }}-A_{0}\right)$ [see Fig. 3(b)], where $A_{0}$ is the small offset. An analogy can be drawn between a nanoscale and a macroscale contact. For macroscale contacts the friction force $F_{\mathrm{f}}$ scales with the true contact area of all contacting asperities, i.e., $F_{\mathrm{f}}=\bar{\tau} \Sigma A_{\text {asp }}$, but it is independent of the apparent contact area $A_{\text {macro }}$.

\section{Applicability of roughness models to nanoscale contacts}

In the previous section the linear dependence of $F_{\mathrm{f}}$ on $L$ has been attributed to the roughness of nanoscale contacts. Here, we provide evidence that roughness theories capture general features of nanoscale contacts. As briefly reviewed in Sec. II, roughness theories predict that the applied load is linear with the true contact area, rather than the nominal contact area. For example, during normal loading of macroscopic contacts, the true contact area $\Sigma A_{\text {asp }}$ is proportional to the applied load $L$, while the nominal contact area $A_{\text {macro }}$ is constant. For nanoscale contacts, the linear relationship between the real contact area $A_{\text {real }}$ and load $L$, i.e., $A_{\text {real }} \propto L$, has been demonstrated in our MD simulations for tip radii of curvature equal to 5, 10, 20, and $30 \mathrm{~nm}$ [see Fig. 4(a)].

Another prediction of roughness theories is that the pressure distribution in contact does not change with load in the regime of small loads..$^{32,37,70-72}$ In our simulations we calculate pressure on individual atoms using a virial theorem. The distribution of normal pressure is calculated for atoms that spend $30 \%$ or more of their time in contact over the simulation time, which is consistent with the criterion we used to define $A_{\text {asp }}$ (see Sec. IV A). The contribution to the pressure 

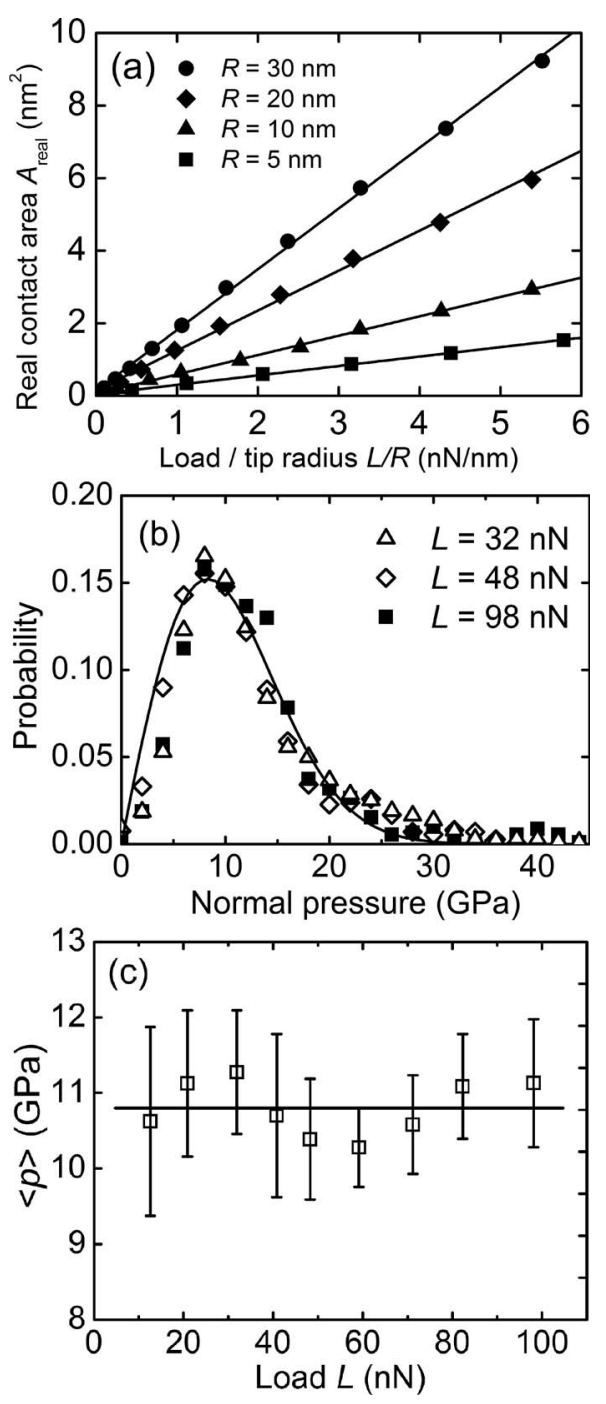

FIG. 4. (a) The real contact area $A_{\text {real }}$ as a function of the applied load $L$ for DLC tips with different tip radii of curvature $R$. The solid lines are linear fits to the MD data. (b) Pressure distribution for atoms in contact during indentation with the $R=30 \mathrm{~nm}$ tip at the applied load of $32 \mathrm{nN}$ (filled squares), $48 \mathrm{nN}$ (empty diamonds), and $98 \mathrm{nN}$ (empty triangles). The distribution histogram is obtained by counting the number of atoms on the sample's surface that is subjected to a given value of normal pressure. The bin size of the histogram is $2 \mathrm{GPa}$. Each pressure distribution is normalized by the total number of atoms in contact (the integral of the pressure distribution is equal to 1). The solid line represents a fit of MD data to Eq. (2.2) obtained at $L=48 \mathrm{nN}$. (c) The fitting parameter $\langle p\rangle$ of a pressure distribution [Eq. (2.2)] plotted as a function of the applied load. Solid line marks the average value of $\langle p\rangle$. The error bar corresponds to the $95 \%$ confidence interval of the fitted values.

distribution from the atoms that are not included in the calculations would be a delta function at near-zero pressure. It is worth pointing out that pressure on all the surface atoms is small but finite due to surface tension even if the surface is out of contact with the tip. Excluding the delta peak at nearzero pressure allows correcting for the effect of surface tension.

We calculated such a pressure distribution at all normal loads and in Fig. 4(b) we show examples for loads of 32, 48, and $98 \mathrm{nN}$. The invariance of pressure distribution with the applied load reflects the fact that the average normal pressure per surface atom is constant, which is equivalent to the fact that $A_{\text {real }}$ is a linear function of $L$.

To demonstrate that the pressure distribution obtained in our simulations can be described by roughness theories of contact behavior, we fit the MD data to the pressure distribution derived of Persson and ${ }^{32-35}$ in the form of Eq. (2.2). The fit of Eq. (2.2) to pressure distribution obtained in MD simulations at a load of $48 \mathrm{nN}$ is plotted as a solid line in Fig. 4(b). The fitting quality $R^{2}$ equals to 0.956 , which shows that the pressure distribution of nanoscale contacts is well represented by roughness theories. Some small deviations from Persson's theory are observed, such as the relatively longer and larger tail of the pressure distribution at larger loads. Campañá et al. ${ }^{70,71}$ showed that such deviations occur in numerically exact calculations of contact of flat rough surfaces. Corrections to Persson's theory haven been proposed by a number of authors. ${ }^{71,73}$

To further test the hypothesis that the pressure distribution does not change with load, in Fig. 4 we plot the fitting parameter $\langle p\rangle$ of Eq. (2.2) as a function of the load. The error bars correspond to $95 \%$ confidence intervals for the fitted values. The value of $\langle p\rangle$ is constant within the error bars of our calculations. In addition, we performed the test of a statistical hypothesis that any two values of $\langle p\rangle$ came from the same distribution. The test showed that a statistical difference cannot be established even for the lowest and the highest values of $\langle p\rangle$. Based on this analysis we can conclude that the pressure distribution in a nanoscale contact is invariant under the applied load in the regime of low loads. In addition, the fact that current roughness theories are based on continuum elasticity theory does not impede their applicability to atomic systems. The role of elasticity theory is to provide a relationship between the contact area and the normal load of the asperities. For atomic systems, the functional dependence of contact area on load may differ from the prediction of continuum elasticity theory but, as we have shown in our study, the general statistical features of atomic roughness are still captured by roughness theories. Given that roughness theories constitute an active area of research and that there are disagreements among quantitative predictions of such theories even for macroscopic contacts, here we focus only on a qualitative agreement between predictions of roughness theories and the behavior of nanoscale contacts.

The linear dependence between $A_{\text {real }}$ and $L$ only applies in the regime where the real contact area is a small fraction of the apparent contact area or in other words where pressure is significant only over the small fraction of the apparent area. Persson et al. showed by numerical analysis that the linear dependence of area on load applies up to the ratio of real contact area over apparent contact area of approximately $20 \% .^{74}$ In our simulations, the ratio of $A_{\text {real }}$ over $A_{\text {asp }}$ is approximately $15-20 \%$, which is within the regime of the applicability of roughness models. In our previous analysis we made an approximation that at the low loads considered in the simulations our single asperity contact can be represented as a flat contact and we compared our results to roughness theories that were derived for flat not spherical contacts. This is a reasonable approximation because even at the highest 

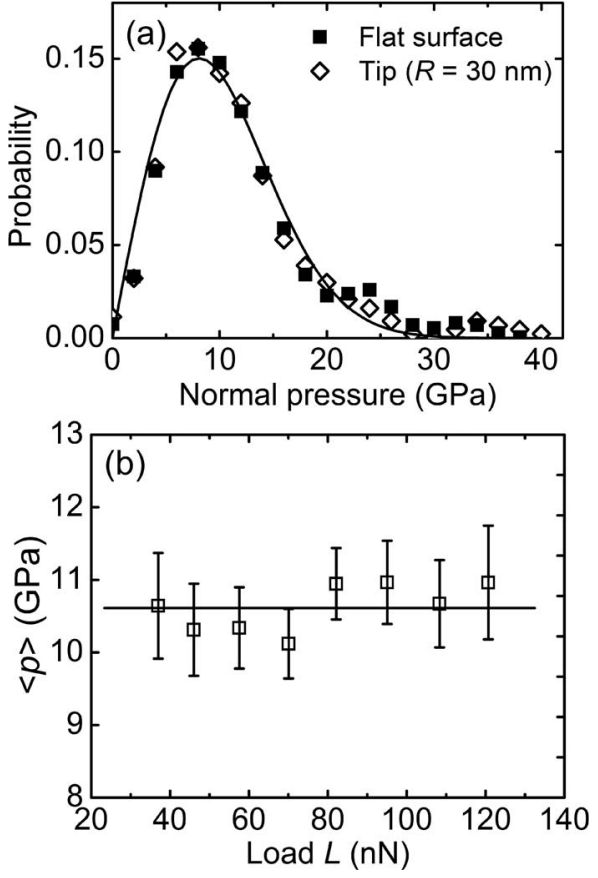

FIG. 5. Pressure distribution in the contact between two flat surfaces. (a) Pressure distribution for atoms in contact. Full squares represent the distribution in the contact between two flat surfaces at load of $70 \mathrm{nN}$; empty diamond symbols represent the pressure distribution in the single asperity contact with tip radius $R=30 \mathrm{~nm}$ at load of $58 \mathrm{nN}$. The solid line is the fit of the MD data for flat surfaces to pressure distribution given by Eq. (2.2). (b) Fitting parameter $\langle p\rangle$ of a pressure distribution [Eq. (2.2)] calculated for flat surfaces and plotted as a function of the applied load. Error bars correspond to the $95 \%$ confidence interval of the fitted values. Solid line corresponds to the average value of $\langle p\rangle$.

normal loads $(98 \mathrm{nN})$ considered in our study, the height variance induced by the curvature of the tip's surface within the contact zone is as small as $2 \AA$, which is lower than the atomic roughness of the interface (e.g., the maximum peakto-valley height is $4.77 \AA$ ). As a direct test of the "flat contact" approximation, we performed additional MD simulations of loading of a flat DLC sample on the same diamond sample as in the case of a spherical tip. The DLC flat surface is prepared by cutting out the center region (approximately $4.4 \times 4.4 \mathrm{~nm}^{2}$ in dimension) of the DLC tip with the curvature radius of $30 \mathrm{~nm}$. The entire tip is curved down to eliminate the radius of curvature. The periodic boundary conditions are applied in both lateral directions to simulate an infinitely large flat surface. The entire system is first relaxed in MD simulations at $300 \mathrm{~K}$. Subsequently, the indentation simulation is performed using the same schedule as described in Sec. III. We find that for the contact of flat surfaces the real contact area is linear with the normal load. The pressure distribution in the contact between flat surfaces is comparable to that of the single asperity contact and both these distributions are shown in Fig. 5(a). Figure 5(b) shows that the pressure distribution in the flat contact is invariant at different loads [compare with data in Fig. 4(c) for a spherical contact]. This analysis shows that in the low load regime the spherical contact can be well approximated as a flat contact

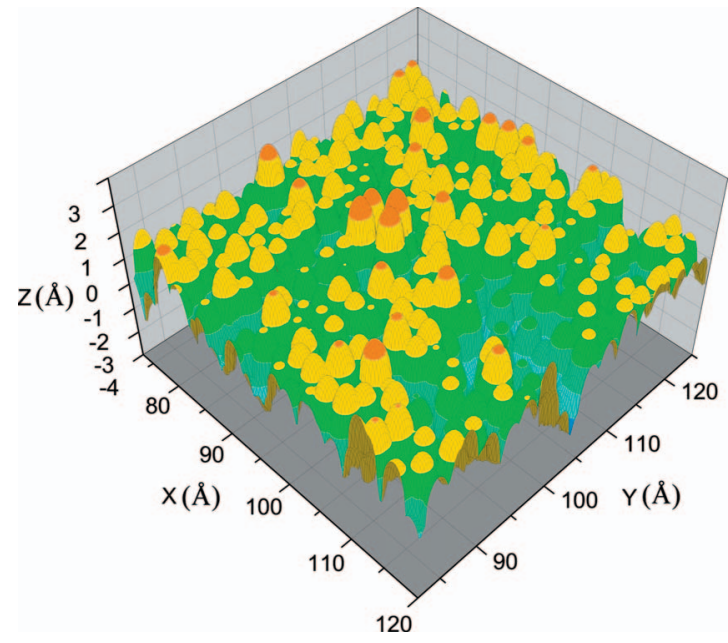

FIG. 6. (Color) Surface height profile of the DLC tip $(R=30 \mathrm{~nm})$. This profile is calculated as the height variance of a methane molecule, when it is being moved over the tip surface under a constant normal force of $1 \mathrm{nN}$.

in order to reproduce the qualitative dependences among $F_{\mathrm{f}}$, $A_{\text {real }}$, and $L$.

The idea of atomic roughness at the nanoscale merits some additional discussion. Quantifying roughness at the atomic scale is more challenging than at larger length scales. One difficulty lies in the fact that atoms are discrete, while most roughness parameters are defined for a continuous surface profile. A straightforward characterization tool is to use the height variance of surface atoms to calculate root-meansquare (rms) roughness. However, this definition has an obvious deficiency as pointed out by Luan and Robbins. ${ }^{11}$ If one considers the case of an atomically flat crystalline surface, the aforementioned definition based on the height variance of atoms gives zero roughness, while the corrugation of the surface potential energy still exists.

By overcoming the deficiency of the above definition, we quantify roughness using a surface height profile obtained by moving an atom or a small molecule over the surface. A similar method has been used by other authors. ${ }^{11}$ The atom or the molecule used to measure roughness is here referred to as a probe. In this method, the measured contour depends on the atomic structure and the composition of the probe used, because interfacial interactions depend on the probe. We use a methane molecule as the probe to measure the surface height profile of the tip surface. The top hydrogen-carbon bond of the methane molecule is vertically aligned toward the tip to closely resemble the geometry of atoms on the $\mathrm{H}$-terminated diamond (111) surface. The probe is moved over a surface and the height of the probe is adjusted to maintain a constant normal force on the probe. The height of the probe at different lateral positions on the surface is plotted and it becomes a surface height profile as shown in Fig. 6 . The constant normal force applied to the probe is chosen to be $1 \mathrm{nN}$, which is close in value to the average force per atom $(0.95 \mathrm{nN})$ calculated for contact atoms in our simulations of SFM experiments.

Once the surface height profile is mapped out, the roughness can be quantified using roughness parameters that are 
conventionally used for engineering surfaces. In addition, the surface height profile is adjusted to eliminate height variance that results from the curvature of the SFM tip. Therefore, the measured roughness parameters represent the roughness that is due solely to the atomic arrangement of atoms on the surface and not to the overall shape of the tip. Based on the surface height profile, we calculated the rms of the height deviation from the average height to be $0.79 \AA$, the maximum peak-to-mean height to be $1.87 \AA$, and the maximum peak-to-valley height to be equal to $4.77 \AA$. For single asperity contact of continuum bodies, Johnson ${ }^{9}$ quantified roughness by taking the ratio of the surface rms roughness and the range of normal displacement of indentation. He showed that as long as this ratio is lower than 0.05 , the roughness-induced deviation of the contact area from predictions of the Hertz model is as small as a few percent. ${ }^{9}$ In our simulation, the total range of the tip's normal displacement is $2.0 \AA$, which corresponds to the maximum load of $98 \mathrm{nN}$. Therefore, the ratio of the rms roughness to tip displacement is 0.4 , which shows that roughness effects in the mechanical behavior of nanoscale contacts are not negligible. We have also calculated a surface height profile by moving the methane probe on a constant potential-energy surface, i.e., the height of the probe was adjusted so to keep the interfacial interaction energy at $0.1 \mathrm{eV}$. Roughness parameters calculated for the surface contour map obtained with this method are all within $5 \%$ of the corresponding parameters calculated for the profile obtained using the constant force method.

By viewing a nanoscale contact as rough, we provide a physical explanation for the linear dependence of $F_{\mathrm{f}}$ on $L$ observed in our MD simulations [see Sec. IV C and Fig. 4(a)]. As discussed in Sec. II, for macroscopic rough contacts this linear $F_{\mathrm{f}}-L$ dependence is traditionally explained by Bowden and Tabor's, GW, or Persson's theory, which all consider the contact to be composed of multiple asperities. Typically, only single asperity continuum models are applied to describe deformation in a single asperity contact and these models predict a sublinear dependence of $F_{\mathrm{f}}$ on $L$. By demonstrating that the concept of roughness can be extended all the way from the macroscale to features as small as atoms, we show that the multiscale roughness theories can be applied to model the mechanical behavior of single asperity contacts. Therefore, the numerous observations ${ }^{14-17,21}$ of the linear $F_{\mathrm{f}^{-}} L$ dependence in nanoscale single asperity contacts can be theoretically explained by our nanoscale roughness picture. Additionally, the idea that a single asperity contact can be understood as a rough contact lays a foundation for constructing a unified roughness theory that encompasses friction properties from macroscopic length scales all the way down to individual atoms.

\section{E. Friction for adhesive contacts}

As discussed in Sec. II, interfacial adhesion plays an important role in the friction behavior of nanoscale contacts. To simulate adhesive contacts, we integrated vdW interactions with the short-range REBO potential as described in Sec. III.

We have demonstrated that the friction force $F_{\mathrm{f}}$ is controlled by the real contact area $A_{\text {real }}$ for nonadhesive contacts
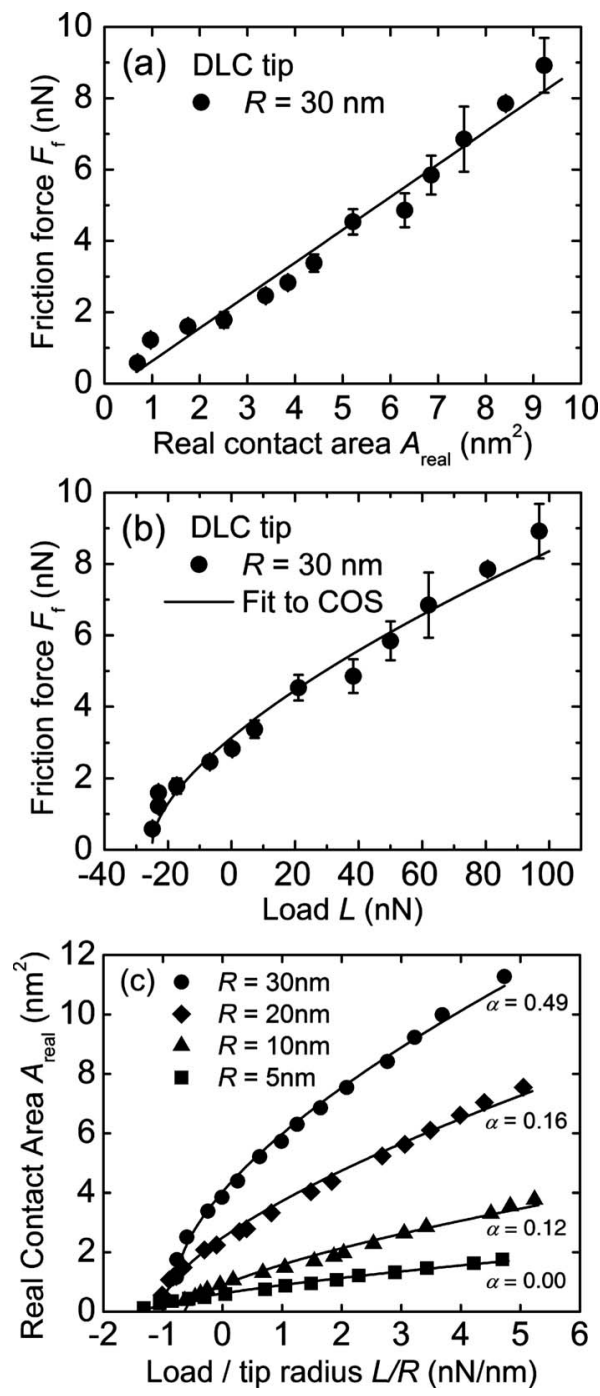

FIG. 7. Adhesive contacts: the friction force $F_{\mathrm{f}}$ as a function of (a) the real contact area $A_{\text {real }}$ and (b) the applied load $L$. Simulations are performed for a DLC tip with radius of curvature $R=30 \mathrm{~nm}$. (c) The real contact area $A_{\text {real }}$ as a function of load $L$ for DLC tips with radii of curvature $5,10,20$, and $30 \mathrm{~nm}$. Solid lines represent (a) a linear fit to MD data and (b),(c) a fit to the COS equation.

(Sec. IV C). Our MD simulations show [Fig. 7(a)] that this relationship holds, i.e., $F_{f}=\tau\left(A_{\text {real }}-A_{0}\right)$, even in the presence of long-range adhesive interactions. This linear dependence of $F_{\mathrm{f}}$ on $A_{\text {real }}$ has been also observed experimentally by Enachescu et al. ${ }^{75,76}$ in their SFM study of sliding friction on hydrogenated diamond (111) surface probed with a platinum tip. During the sliding experiments, the friction force, the interfacial electrical conductance, and the normal load were measured simultaneously. If $A_{\text {real }} \neq A_{\text {asp }}$, then the electrical conductance is proportional to the real contact area $A_{\text {real }}$ rather than to the asperity contact area $A_{\text {asp }}$ defined by the edge of the contact zone. If $A_{\text {real }}=A_{\text {asp }}$, then the electrical conductance is a good measure of both of these areas. In either case, the experimentally observed linear dependence of the friction force on the electrical conductance is equivalent to the linear dependence of $F_{\mathrm{f}}$ on $A_{\text {real }}$ observed in our MD simulations. 
The fact that $F_{\mathrm{f}}$ is linear with the mean number of atoms in contact $\left\langle N_{\text {at }}\right\rangle$ for both nonadhesive and adhesive contacts demonstrates that friction is controlled by the short-range chemical interactions. We have also shown that pressure distribution in nanoscale contacts is consistent with roughness theories. Consequently, both the pressure distribution and the mean number of atoms in contact $\left\langle N_{\text {at }}\right\rangle$ are fundamental quantities that capture the behavior of nanoscale contacts. The real contact area $A_{\text {real }}$ has been derived from the definition of $\left\langle N_{\text {at }}\right\rangle$ and it is useful for drawing analogies to contacts at larger length scales. It is worth pointing out that the observation that $F_{\mathrm{f}}$ is linear with $\left\langle N_{\mathrm{at}}\right\rangle$ is more general than the definition of $A_{\text {real }}$. For instance, in a recent MD study ${ }^{77}$ we have investigated friction of H-terminated DLC tip on a diamond surface with varying $\mathrm{H}$ coverage. We found that shear strength (and therefore also friction) was linear with surface coverage or, in other words, with the number of covalent $\mathrm{C}-\mathrm{H}$ bonds formed across the interface during sliding. This result suggests that the linear relationship between $F_{\mathrm{f}}$ and $\left\langle N_{\text {at }}\right\rangle$ is applicable to adhesive chemical bonds as well as to the purely repulsive $\mathrm{H}-\mathrm{H}$ bonds considered in the current study.

We have shown in Secs. IV B and IV C that roughness theories describe the frictional behavior of nonadhesive contacts, i.e., $F_{\mathrm{f}}$ (and $A_{\text {real }}$ ) depends linearly on $L$. Therefore, for adhesive contacts, the applicability of adhesive continuum models to the dependence of $F_{\mathrm{f}}$ (and $A_{\text {real }}$ ) on $L$ is not expected. In contrast, our MD simulations results show that both the friction force $F_{\mathrm{f}}$ and the real contact area $A_{\text {real }}$ are sublinear functions of $L$ as shown in Figs. 7(b) and 7(c). This qualitative agreement of the dependence of both $F_{\mathrm{f}}$ and $A_{\text {real }}$ on $L$ between our MD simulations and continuum models of adhesive singe asperity contacts is surprising.

To test whether there is quantitative agreement between the simulated nanoscale contacts and continuum theories, we fit our simulation data to the Maugis-Dugdale model. ${ }^{42} \mathrm{Be}-$ cause continuum theories provide a relationship between contact area and the applied load, in fitting continuum models to the friction force-load dependence one assumes that the friction force is linear with the contact area. This assumption is frequently used in experimental studies because the friction force is easier to measure than the contact area. Furthermore, because the Maugis-Dugdale model lacks a simple analytical expression relating the contact area (and the friction force $F_{\mathrm{f}}$ ) and the normal load $L$, we use an interpolation formula developed by Carpick, Ogletree, and Salmeron (COS). ${ }^{78}$ The COS equation has been widely used to interpret experimental data and has been physically justified by Schwarz. ${ }^{45}$ From fitting of the $F_{\mathrm{f}}-L$ curve, we determine the COS transition parameter $\alpha$ to be equal to 0.21 . This value of $\alpha$ corresponds to the Tabor parameter $\mu_{\mathrm{T}}=0.19$. Based on formula (2.5), we calculated the range of theoretical values of the Tabor parameter $\mu_{\mathrm{T}}$ for our simulated contact. In the calculation, we use the value of $R=30 \mathrm{~nm}$ (known from the setup of our model system) and $E^{*}=303 \mathrm{GPa}$ (Sec. IV B). The interface separation distance $z_{0}$ is chosen as $1.7 \AA$ for the estimate of the lower limit of $\mu_{\mathrm{T}}$, and it is equal to the range of $\mathrm{H}-\mathrm{H}$ interaction in the REBO potential. To estimate the upper limit of $\mu_{\mathrm{T}}, z_{0}$ is chosen to be $1.0 \AA$, which corresponds to the atomic radius of a hydrogen atom. The value of $\gamma$ is calculated from the pull-off force measured in our simulation by the method specified in Ref. 78. Using Eq. (2.5), we estimate the range of $\mu_{\mathrm{T}}$ to be between 0.11 and 0.22 . The Tabor parameter obtained from fitting our simulation data to the Maugis-Dugdale model $\mu_{\mathrm{T}}=0.19$ falls into the range of the theoretically calculated values. Based on this analysis, it appears that continuum models for adhesive contacts quantitatively agree with the friction behavior obtained in our MD simulations.

The consistency between the simulation results and the Maugis-Dugdale model is also reflected in the dependence of the real contact area $A_{\text {real }}$ on the applied load $L$. Figure 7(c) shows data obtained from MD simulations of normal loading of SFM tips with various radii of curvature. For all the tip radii considered, the functional dependence of $A_{\text {real }}$ on $L$ is well described by the COS equation. The COS transition parameter $\alpha$, which is estimated from the fitting, equals 0.49 , $0.16,0.12$, and 0.00 , for tip curvature radii $R=30,20,10$, and $5 \mathrm{~nm}$, respectively. The transition parameter $\alpha$ decreases with decreasing $R .^{79}$ This trend is consistent with continuum theories, which predict that as the tip radius decreases, the Tabor parameter decreases and the contact behavior transitions from the JKR to the DMT limit. ${ }^{42-44}$ Indeed, in our simulations the $F_{\mathrm{f}}-L$ dependence reaches the DMT limit $\left(\alpha=0.0\right.$ and $\left.\mu_{\mathrm{T}}=0.0\right)$ for the tip $R=5 \mathrm{~nm}$.

Our simulation data show also a very good agreement with the contact pressures and shear strengths estimated in the SFM experiment. ${ }^{6}$ Using the fitting procedure described in Ref. 6, the interfacial shear strength $\tau$ estimated from our simulation data is $508 \mathrm{MPa}$, whereas the experimental values for the same material system fall into the range 201-485 $\mathrm{MPa}$. The experimental contact pressure estimated for the normal load of $100 \mathrm{nN}$ is in the range $1.8-4.3 \mathrm{GPa}$, whereas at the same load the normal pressure calculated directly in our simulation is $6.1 \mathrm{GPa}$. This slightly larger contact pressure in the simulations is expected given that the curvature radius of the simulated tip was slightly smaller $(R=30 \mathrm{~nm})$ than the size of the tip used in the SFM experiment $(R=45 \mathrm{~nm})$. Our simulation results seem to be in an agreement not only with continuum models but also with values measured and estimated in SFM experiments.

In order to test whether continuum models truly describe the correct physics of nanoscale contacts in the presence of adhesion, we separate the total load into the contributions that come from the elastic restoring force $L_{\mathrm{el}}$ and from the vdW attraction, i.e., $L=L_{\mathrm{el}}+L_{\mathrm{vdW}}$. The elastic restoring force $L_{\mathrm{el}}$ results from the elastic deformation of the contact. The contribution $L_{\mathrm{vdW}}$ represents the interfacial adhesion (i.e., $\mathrm{vdW}$ forces). The value of $L_{\mathrm{el}}$ can be calculated by subtracting the vdW force $L_{\mathrm{vdW}}$ from the total load $L$. If the deformation of an adhesive contact follows Hertzian mechanics, then the asperity contact area $A_{\text {asp }}$ will scale with the elastic restoring force as $L_{\mathrm{el}}^{2 / 3} \cdot{ }^{45} \mathrm{As}$ indicated by open symbols in Fig. 8(a), the dependence of $A_{\text {asp }}$ on $L_{\mathrm{el}}$ is indeed sublinear and it shows a qualitative agreement with the Hertz model (solid line). However, the effective modulus $E^{*}$ calculated by fitting MD data to the Hertz model is $198 \mathrm{GPa}$, which is $35 \%$ lower than the effective modulus calculated directly from theory. This analysis confirms that both in nonadhesive and in adhesive contacts continuum mechanics qualitatively de- 

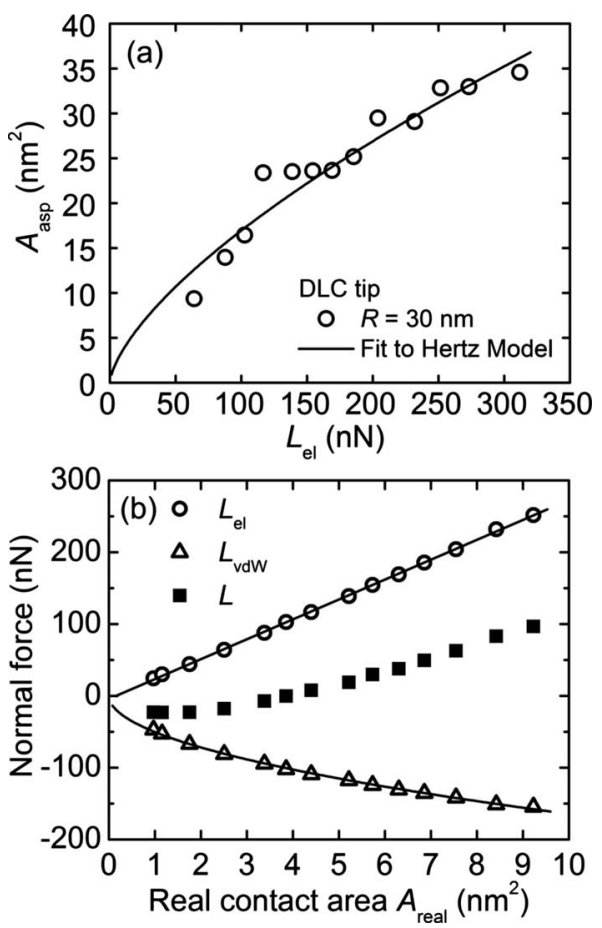

FIG. 8. (a) The dependence of asperity contact area on the elastic restoring force $L_{\mathrm{el}}$. Symbols correspond to data obtained from simulations and the solid line represents a fit to Hertz model. (b) Contributions to the total load $L$ (full squares) as a function of the real contact area $A_{\text {real }}$. The total load $L$ is a sum of contributions from the vdW interactions $L_{\mathrm{vdW}}$ (empty triangles) and the elastic restoring force $L_{\mathrm{el}}$ (empty circles).

scribes the dependence of the asperity area $A_{\text {asp }}$ on load, but it shows large quantitative deviations.

We also tested the applicability of roughness theories to describe deformation in nanoscale adhesive contacts. If atomic roughness controls deformation of the contact, then the real contact area $A_{\text {real }}$ will scale linearly with $L_{\mathrm{el}}$. Figure 8(b) shows the dependence of $A_{\text {real }}$ on $L, L_{\mathrm{vdW}}$, and $L_{\mathrm{el}}$. Indeed, the dependence of $A_{\text {real }}$ on $L_{\mathrm{el}}$ is linear (empty circles), which demonstrates that the adhesive contact behaves as a rough interface and the contact is in the regime where the fraction $A_{\text {real }} / A_{\text {asp }}$ is small. This observation is consistent with the fact that kinetic friction $F_{\mathrm{f}}$ scales linearly with $A_{\text {real }}$ and not with $A_{\text {asp }}$ [Fig. 7(a)]. Consequently, while both continuum mechanics and roughness theories qualitatively describe the dependence of appropriate contact areas on the applied load, it is the roughness theory that correctly captures the frictional behavior of the contact. Roughness theories predict that, when the pressure distribution is significant only over a small fraction of the apparent contact area, it is the real contact area that controls the mechanical behavior of the contact.

The question remains why the dependence of $A_{\text {real }}\left(\right.$ or $\left.F_{\mathrm{f}}\right)$ on the total normal load $L$ is sublinear [Figs. 7(c) and 8(b)]. We have shown that $A_{\text {real }}$ is linear with $L_{\text {el }}$ [circles in Fig. 8(b)]. The nonlinear contributions to $A_{\text {real }}$ (or $F_{\mathrm{f}}$ ) come from $L_{\mathrm{vdW}}$ [triangles in Fig. 8(b)]. The dependence of $A_{\text {real }}$ on $L$ is a combined dependence of a linear dependence $A_{\text {real }}$ on $L_{\mathrm{el}}$ and a nonlinear dependence $A_{\text {real }}$ on $L_{\mathrm{vdW}}$, which explains why $A_{\text {real }}$ scales sublinearly with $L$ [squares in Fig. 8(b)]. This analysis demonstrates that, as the interfacial adhesion is increased, a transition takes place from linear to sublinear dependences of $F_{\mathrm{f}}$ on $L$ and $A_{\text {real }}$ on $L$.

Since frictional behavior is controlled by the real contact area $A_{\text {real }}$ and roughness theories apply to these contacts, how can one explain the fact that the Maugis-Dugdale model quantitatively describes our simulation data [i.e., the dependence of $F_{\mathrm{f}}$ on $L$ shown in Fig. 7(b)] so well? The good quality of the fits results from the flexibility of the MaugisDugdale model, i.e., three degrees of freedom in the COS equation. Once we considered only the elastic contribution $L_{\mathrm{el}}$ to the normal load and tested its agreement with continuum theories, the quantitative deviations between continuum theory and our modeled data became apparent. We show that while fitting to continuum contact models may be a convenient way to compare results between different studies (e.g., between simulations and experiments), it is the roughness theories that correctly capture the frictional behavior of nanoscale contacts.

\section{F. Linear to sublinear transition of friction-load dependence}

Both linear and sublinear dependences of $F_{\mathrm{f}}$ on $L$ have been reported in MD simulations and it is instructive to compare our results to those from other simulations to determine where the apparent discrepancies come from. One source of differences comes from the different surface roughness of the modeled systems. For instance, Luan and Robbins ${ }^{11}$ observed that for the contact of an amorphous tip with a crystalline sample the dependence of the friction force on the load is sublinear, i.e., $F_{\mathrm{f}} \propto L^{2 / 3}$, which is in contrast to the linear dependence observed in our study. The different surface chemistry of our system, i.e., the termination with adsorbate atoms, may also contribute to the higher surface roughness than the one in Ref. 11. Specifically, roughness parameters reported in Ref. 11 indicate a relatively low roughness of the modeled system, i.e., the maximum height variance of the amorphous tip was reported as $3 \AA$, whereas in our system the equivalent quantity (i.e., the peak-to-valley height calculated from the surface contour map) equals $4.77 \AA$ (Sec. IV C).

One can expect that lower surface roughness will result in a better agreement of contact behavior with continuum mechanics models, which may explain why Ref. 11 reported $F_{\mathrm{f}} \propto A_{\text {asp }}$ (and $F_{f}$ being sublinear with $L$ ) in contrast to our simulations where $F_{\mathrm{f}}$ scales linearly with $A_{\text {real }}$ (and $F_{\mathrm{f}}$ is a linear function of $L$ ). Our study shows that friction scales differently with $A_{\text {real }}$ and with $A_{\text {asp }}$ for atomically rough single asperity contacts. In the limit of smooth surfaces, where continuum mechanics quantitatively describes contact behavior, the friction force at a single asperity has been often observed $^{8}$ to be proportional to the asperity contact area, i.e., $F_{\mathrm{f}} \propto A_{\text {asp }}$, which is seemingly in contradiction with our general conclusions that in dry nanoscale contacts $F_{\mathrm{f}}$ scales linearly with $A_{\text {real }}$. This apparent contradiction can be resolved by realizing that when the contact becomes smoother, $A_{\text {real }}$ converges to $A_{\text {asp }}$ because more atoms within the contact zone come into real contact with the counterface. Conse- 
quently for contacts with sufficiently small roughness, the friction force $F_{\mathrm{f}}$ will be linear with both $A_{\text {asp }}$ and $A_{\text {real }}$ and the real contact area $A_{\text {real }}$ remains a well-defined quantity regarding of the contact roughness.

Our MD simulations and analysis show that as the roughness of the so-called single asperity contact is decreased, a transition takes place from a linear to a sublinear $F_{\mathrm{f}^{-}} L$ dependence. Such a transition has been observed by Reitsma et $a l .{ }^{13}$ in their SFM experiment on $\mathrm{Si}$, where the surface was smoothened during sliding (i.e., roughness was decreased) and a linear dependence of the friction force on the load transitioned to a sublinear dependence. Our study also demonstrates a transition from linear to sublinear $F_{\mathrm{f}}$ vs $L$ dependence as a result of an increased interfacial adhesion. An experimental example of such a transition comes from the SFM study of ultrananocrystalline diamond by Grierson, ${ }^{16}$ who reported a sublinear dependence in experiments on an unpassivated sample (i.e., with high adhesion) and a linear dependence for samples passivated with hydrogen atoms (i.e., with low adhesion).

The linear to sublinear transition of the $F_{\mathrm{f}}-L$ dependence is therefore controlled by two factors: roughness and interfacial adhesion. In the light of this transition, we can understand that friction forces in the load- and adhesion-controlled regimes are in fact related to the same physical phenomenon. The fundamental quantity that governs friction in both cases is the number of atoms in contact or a derived quantity of the real contact area $A_{\text {real }}$. One consequence of our study is the fact that the friction force $F_{\mathrm{f}}$ is linear with the real contact area at any length scale as long as the real contact area is correctly defined at that length scale. The transition from load-controlled friction to adhesion-controlled friction is governed by the interplay between adhesion and roughness. As shown in Sec. IV B, when the roughness of contact is large and the interfacial adhesion is low, friction is load controlled, i.e., the friction force $F_{\mathrm{f}}$ is a linear function of load $L$. On the other hand, friction is adhesion controlled for contacts with low roughness and/or high adhesion. Further studies are needed to quantify the transition from linear to sublinear behavior in terms of the roughness parameters and the strength of adhesion. Our findings are consistent with the phenomenologically determined rules that friction of smooth undamaged surfaces is adhesion controlled and that of rough damaged surfaces is load controlled. ${ }^{12}$

\section{DISCUSSION AND CONCLUSION}

Before concluding it is yet instructive to explicitly discuss the discrepancy in the sliding velocities used in our MD simulations $(20 \mathrm{~m} / \mathrm{s})$ and in typical SFM experiments (on the order of $\sim \mu \mathrm{m} / \mathrm{s}$ ). While it would certainly be advantageous to carry out simulations on time scales comparable to SFM experiments, such simulations are not possible using molecular and atomistic simulations (except for few very specific cases where accelerated molecular-dynamics techniques can be used $\left.{ }^{55,56}\right)$. However, we argue that the high sliding velocity used in our simulations has little effect on the magnitude of friction and it does not change the observed friction laws. First of all, the sliding velocity in our simulations is much smaller (600-900 times) than the sound velocity of diamond, which ensures that the heat generated in contact during sliding is efficiently dissipated. Second, each surface atom vibrates approximately 1000 times during when the tip moves by one lattice constant of the surface lattice, which means that the system has enough time to relax during sliding. The above arguments are also supported by the fact that the temperature gradient observed in our simulation during sliding is negligible. Additionally, we have performed a direct test of the effect of the sliding velocity on friction, i.e., we performed separate simulations with sliding velocities of $v=40$ and $2 \mathrm{~m} / \mathrm{s}$. We found that for sliding at a given normal displacement of the tip, both the average contact area and the average normal load are constant at all velocities considered. Also, only small change in the friction force is observed in this regime, i.e., $\Delta F_{\mathrm{f}} / \Delta v \approx 0.02 \mathrm{nN} /(\mathrm{m} / \mathrm{s})$. Therefore, it is reasonable to assume that the mechanisms underlying friction in our study depend weakly on sliding velocity, which enables a comparison between results of MD simulations and SFM experiments.

We can also ask about the generality of our models and conclusions. Since we have demonstrated that roughness theories describe correctly the mechanical behavior of nanoscale contacts, consequently, assumptions of roughness theories impose certain limitations on the applicability of our model. For instance, we have emphasized in this paper that roughness theories apply when $A_{\text {real }} / A_{\text {asp }}$ is small or in other words when the roughness is large. This condition is satisfied for H-passivated DLC surface investigated in our study; however, roughness may decrease when surfaces are not passivated or when there is interaction among the adsorbate atoms. If such changes in surface chemistry lead to a sufficient reduction in surface corrugation (shown in Fig. 6), so that $A_{\text {real }}$ converges to $A_{\text {asp }}$, then one can expect to observe both a sublinear behavior of $F_{\mathrm{f}}$ on $L$ and a linear behavior of $F_{\mathrm{f}}$ on $A_{\text {asp. }}$. Another assumption of roughness theories that leads to a linear dependence of $F_{\mathrm{f}}$ on $L$ is that the normal loads are low (low enough so that $A_{\text {real }} \ll A_{\text {asp }}$ ). In our case we observed the linear dependence for loads up to $\sim 300 \mathrm{nN}$. The geometry of contact also needs to be taken into consideration. The geometry considered in our study is consistent with those of single asperity contacts where the tip can be approximated by a spherical cap near the contact zone and where the roughness is random. These conditions are typically satisfied by engineering surfaces. On the other hand, our model may be not applicable to some special contact geometries, such as a commensurate contact between two crystalline solids or other interfaces that lack randomness (e.g., graphite flakes on a surface ${ }^{80}$ ), as well as cases where the edge of the contact contributes significantly to the friction force (e.g., sliding of small islands across a flat surface $\left.^{81}\right)$.

It is also instructive to compare our results to earlier MD simulations and in particular to those reported by Luan and Robbins ${ }^{10}$ who used geometry and size of SFM tips comparable to the ones in our simulations. One major difference between these two studies is that the surfaces investigated in Ref. 10 were smoother than ours, which is evidenced in the roughness parameters discussed in Sec. IV F. For systems with such a low surface roughness the friction force $F_{\mathrm{f}}$ was 
found to be linear with the area $A_{\text {asp }}$ defined by the edge of the contact zone and $F_{\mathrm{f}}$ was a sublinear function of load $L$. More recently the same group ${ }^{63}$ has shown that for such smooth surfaces $A_{\text {real }}$ has the same sublinear scaling with $L$ as does $A_{\text {asp }}$, which is expected if the ratio $A_{\text {real }}$ approaches $A_{\text {asp. }}$. On the other hand, our study shows that surface roughness of H-terminated surfaces is considerably larger than that of bare surfaces and that this increase in roughness qualitatively changes the dependence of the friction force $F_{\mathrm{f}}$ on the contact area. In our case, $F_{\mathrm{f}}$ is no longer linear with $A_{\text {asp }}$, but instead it is linear with the real contact area $A_{\text {real }}$. Our MD study of SFM on dry surfaces considers the limit where $A_{\text {real }}$ is small as compared to $A_{\text {asp }}$ (less than 20\%). We demonstrate that in such a limit these two areas scale differently with the applied load $L$. Specifically while $A_{\text {asp }}$ is still sublinear with $L$, as predicted by continuum mechanics, $A_{\text {real }}$ is now linear with load, as predicted by roughness theories, and it is $A_{\text {real }}$ that controls friction.

Cheng et al. ${ }^{63}$ pointed out that at least for smooth surfaces, i.e., where $A_{\text {real }} / A_{\text {asp }}$ is large, the transition from linear to sublinear dependence of $F_{\mathrm{f}}$ on $L$ can take place if one considers static friction instead of kinetic friction. This is an interesting point and its applicability to the regime of rough contacts, i.e., where $A_{\text {real }} / A_{\text {asp }}$ is small, needs to be tested. In our study, we do not have enough data to test this hypothesis due to the computational intensity of MD simulations that involve the REBO potential. The focus of our study is to explain different friction laws observed in SFM experiments and therefore we report kinetic friction (which is measured in experiments) and we consider the regime of loads typical for wearless SFM experiments, i.e., $L \leq 100 \mathrm{nN}$. We found that in nonadhesive contacts the kinetic friction force is linear with the load. It is worth pointing out that the magnitude of the friction force depends not only on the interfacial properties, but also on the lateral stiffness of the contact. Cheng et $a l .{ }^{63}$ performed a series of MD simulations with a simplified Lennard-Jones potential and showed that the linearity of the kinetic friction force on load persists independently of the lateral contact stiffness.

In summary, we employed large-scale MD simulations to determine friction laws in dry and wearless nanoscale contacts, which laws are summarized in Table I. By using accurate interatomic potentials and performing MD simulations at length scales comparable to experiments, we were able to achieve a quantitative agreement between our simulation results and data obtained in SFM experiments on the same materials systems. We have determined how the friction force $F_{\mathrm{f}}$ is related to the normal load $L$, the asperity contact area $A_{\text {asp }}$ (defined by the edge of the contact zone), and the real contact area $A_{\text {real }}$ (defined by the number of atoms that interact across the contact interface). Our simulation results confirmed that while continuum contact mechanics works qualitatively at the nanoscale, i.e., $A_{\text {asp }}$ scales with load approximately as $L^{2 / 3}$, there are significant quantitative deviations between the simulations and the continuum theories. We showed that a fundamental quantity that controls friction is the number $\left\langle N_{\text {at }}\right\rangle$ of atoms in contact, i.e., the number of atoms that interact across the contact interface. If the real contact area $A_{\text {real }}$ is defined in terms of $\left\langle N_{\mathrm{at}}\right\rangle$, then $F_{\mathrm{f}}$ is linearly dependent on this contact area, in analogy to relationships observed at larger length scales (see Table I). We demonstrated that in rough nanoscale contacts friction behavior is well captured by roughness theories. Our study lays a foundation for developments of a unified roughness theory that extends from macroscale all the way down to single atoms. We have also shown that a transition from a linear to sublinear dependence of the friction force on the load is controlled by the interplay between roughness and adhesion in the contact. Our roughness picture of a nanoscale contact provides a consistent explanation for the experimental observations of the transition. It also allows resolving some of the controversies that exist among MD simulations regarding the dependence of the friction force on the load.

What is still needed is a quantification of the linear to sublinear dependence in terms of surface roughness, adhesion range and strength, tip geometry, range of normal loads, etc., so that one can predict the conditions when such a transition would take place. Also, the proposed friction laws apply to purely elastic, wearless, and dry contacts. To describe friction behavior of engineering surfaces, other factors need to be included, e.g., plasticity and lubrication. We have shown that roughness theories qualitatively describe behavior of nanoscale contacts. In order to build quantitative models, modifications of roughness theories may be required for the dependence of the contact area on the load of atomic-size asperities and/or for the coupling between deformations of these asperities, because current roughness theories rely on continuum mechanics to describe these phenomena at larger length scales.

\section{ACKNOWLEDGMENT}

We gratefully acknowledge financial support from the American Chemical Society Grant No. PRF-47978-G5.
${ }^{1}$ A. D. Romig, Acta Mater. 51, 5837 (2003).

${ }^{2}$ F. W. DelRio, M. P. De Boer, J. A. Knapp, E. D. Reedy, P. J. Clews, and M. L. Dunn, Nature Mater. 4, 629 (2005).

${ }^{3}$ G. Amontons, Mem. de l'Academie Royale A, 275 (1699).

${ }^{4}$ R. W. Carpick and M. Salmeron, Chem. Rev. 97, 1163 (1997).

${ }^{5}$ I. Szlufarska, M. Chandross, and R. W. Carpick, J. Phys. D: Appl. Phys. 41, 123001 (2008).

${ }^{6}$ G. T. Gao, R. J. Cannara, R. W. Carpick, and J. A. Harrison,
Langmuir 23, 5394 (2007).

${ }^{7}$ E. Riedo, J. Chevrier, F. Comin, and H. Brune, Surf. Sci. 477, 25 (2001).

${ }^{8}$ J. N. Israelachvili and A. D. Berman, in Handbook of Micro/ Nanotribology, 2nd ed., edited by B. Bhushan (CRC Press, Boca Raton, FL, 1999), p. 371.

${ }^{9}$ K. L. Johnson, Contact Mechanics (Cambridge University Press, Cambridge, England, 1987). 
${ }^{10}$ B. Luan and M. O. Robbins, Nature (London) 435, 929 (2005).

${ }^{11}$ B. Luan and M. O. Robbins, Phys. Rev. E 74, 026111 (2006).

${ }^{12}$ J. Gao, W. D. Luedtke, D. Gourdon, M. Ruths, J. N. Israelachvili, and U. Landman, J. Phys. Chem. B 108, 3410 (2004).

${ }^{13}$ M. G. Reitsma, R. G. Cain, S. Biggs, and D. W. Smith, Appl. Surf. Sci. 252, 4964 (2006).

${ }^{14}$ M. Ruths, N. A. Alcantar, and J. N. Israelachvili, J. Phys. Chem. B 107, 11149 (2003).

${ }^{15}$ M. Ruths, Langmuir 19, 6788 (2003).

${ }^{16}$ D. S. Grierson, Ph.D. thesis, University of Wisconsin, Madison, 2008.

${ }^{17}$ T. J. Colburn and G. J. Leggett, Langmuir 23, 4959 (2007).

${ }^{18}$ F. P. Bowden and D. Tabor, Friction and Lubrication of Solids (Wiley, New York, 1956).

${ }^{19}$ F. P. Bowden and D. Tabor, Friction and Lubrication of Solids Part I (Oxford University Press, New York, 1954); Friction and Lubrication of Solids Part II (Oxford University Press, New York, 1964).

${ }^{20}$ L. Wenning and M. H. Müser, EPL 54, 693 (2001).

${ }^{21}$ M. Chandross, C. D. Lorenz, M. Stevens, and G. S. Grest, Langmuir 24, 1240 (2008).

${ }^{22}$ Y. Mo, K. Turner, and I. Szlufarska, Nature (London) 457, 1116 (2009).

${ }^{23}$ F. P. Bowden and D. Tabor, Nature (London) 150, 197 (1942).

${ }^{24}$ F. P. Bowden and D. Tabor, Proc. R. Soc. London, Ser. A 169, 391 (1939)

${ }^{25}$ J. A. Greenwood and J. B. P. Williamson, Proc. R. Soc. London, Ser. A 295, 300 (1966).

${ }^{26}$ A. W. Bush, R. D. Gibson, and T. R. Thomas, Wear 35, 87 (1975).

${ }^{27}$ A. Kapoor and K. L. Johnson, Proc. R. Soc. London, Ser. A 445, 367 (1994)

${ }^{28}$ K. N. G. Fuller and D. Tabor, Proc. R. Soc. London, Ser. A 345, 327 (1975)

${ }^{29}$ D. Maugis, J. Adhes. Sci. Technol. 10, 161 (1996).

${ }^{30}$ M. Ciavarella, J. A. Greenwood, and M. Paggi, Wear 265, 729 (2008).

${ }^{31}$ J. A. Greenwood and J. J. Wu, Meccanica 36, 617 (2001).

${ }^{32}$ B. N. J. Persson, J. Chem. Phys. 115, 3840 (2001).

${ }^{33}$ B. N. J. Persson, Phys. Rev. Lett. 87, 116101 (2001).

${ }^{34}$ B. N. J. Persson, Phys. Rev. Lett. 99, 125502 (2007).

${ }^{35}$ B. N. J. Persson, F. Bucher, and B. Chiaia, Phys. Rev. B 65 , 184106 (2002).

${ }^{36}$ W. Manners and J. A. Greenwood, Wear 261, 600 (2006).

${ }^{37}$ S. Hyun, L. Pei, J. F. Molinari, and M. O. Robbins, Phys. Rev. E 70, 026117 (2004).

${ }^{38}$ G. Binnig, C. F. Quate, and C. Gerber, Phys. Rev. Lett. 56, 930 (1986).

${ }^{39}$ H. Hertz, J. Reine Angew. Math. 92, 156 (1881).

${ }^{40}$ K. L. Johnson, K. Kendall, and A. D. Roberts, Proc. R. Soc. London, Ser. A 324, 301 (1971).

${ }^{41}$ B. V. Derjaguin, V. M. Muller, and Y. P. Toporov, J. Colloid Interface Sci. 53, 314 (1975).

${ }^{42}$ D. Maugis, J. Colloid Interface Sci. 150, 243 (1992).

${ }^{43}$ D. Tabor, J. Colloid Interface Sci. 58, 2 (1977).

${ }^{44}$ K. L. Johnson and J. A. Greenwood, J. Colloid Interface Sci. 192, 326 (1997).

${ }^{45}$ U. D. Schwarz, J. Colloid Interface Sci. 261, 99 (2003).

${ }^{46}$ R. G. Horn, J. N. Israelachvili, and F. Pribac, J. Colloid Interface Sci. 115, 480 (1987)
${ }^{47}$ D. Gourdon and J. N. Israelachvili, Phys. Rev. E 68, 021602 (2003).

${ }^{48}$ A. Berman, C. Drummond, and J. Israelachvili, Tribol. Lett. 4, 95 (1998).

${ }^{49}$ M. P. Allen and D. J. Tildesley, Computer Simulations of Liquids (Clarendon Press, Oxford, 1987).

${ }^{50}$ A. Socoliuc, R. Bennewitz, E. Gnecco, and E. Meyer, Phys. Rev. Lett. 92, 134301 (2004).

${ }^{51}$ R. W. Carpick, Ph.D. thesis, University of California, Berkeley, 1997.

${ }^{52}$ M. H. Müser, L. Wenning, and M. O. Robbins, Phys. Rev. Lett. 86, 1295 (2001).

${ }^{53}$ K. L. Johnson, Proc. R. Soc. London, Ser. A 453, 163 (1997).

${ }^{54}$ I. Szlufarska, Mater. Today 9, 42 (2006).

${ }^{55}$ A. Martini, Y. Dong, D. Perez, and A. Voter, Tribol. Lett. 36, 63 (2009).

${ }^{56}$ A. F. Voter, Phys. Rev. B 57, R13985 (1998).

${ }^{57}$ G. He, M. H. Müser, and M. O. Robbins, Science 284, 1650 (1999).

${ }^{58}$ D. Brenner, O. A. Shenderova, J. A. Harrison, S. J. Stuart, B. Ni, and S. B. Sinnott, J. Phys.: Condens. Matter 14, 783 (2002).

${ }^{59}$ G. T. Gao, P. T. Mikulski, and J. A. Harrison, J. Am. Chem. Soc. 124, 7202 (2002).

${ }^{60}$ S. B. Sinnott, R. J. Colton, C. T. White, O. A. Shenderova, D. W. Brenner, and J. A. Harrison, J. Vac. Sci. Technol. A 15, 936 (1997).

${ }^{61}$ S. J. Stuart, A. B. Tutein, and J. A. Harrison, J. Chem. Phys. 112, 6472 (2000).

${ }^{62}$ J. A. Greenwood, Proc. R. Soc. London, Ser. A 453, 1277 (1997).

${ }^{63}$ S. Cheng, B. Luan, and M. Robbins, Phys. Rev. E 81, 016102 (2010).

${ }^{64}$ C. Yang and B. N. J. Persson, Phys. Rev. Lett. 100, 024303 (2008).

${ }^{65}$ B. Q. Luan and M. O. Robbins, Tribol. Lett. 36, 1 (2009).

${ }^{66}$ J. J. Vlassak and W. D. Nix, J. Mech. Phys. Solids 42, 1223 (1994).

${ }^{67}$ J. J. Vlassak and W. D. Nix, Philos. Mag. A 67, 1045 (1993).

${ }^{68}$ C. Zuiker, A. R. Krauss, D. M. Gruen, X. Pan, J. C. Li, R. Csencsits, A. Erdemir, C. Bindal, and G. Fenske, Thin Solid Films 270, 154 (1995).

${ }^{69}$ T. Takeno, T. Komoriya, I. Nakamori, H. Miki, T. Abe, T. Uchimoto, and T. Takagi, Tribological properties of partly polished diamond coatings (Elsevier Science, New York, 2005), p. 2118.

${ }^{70}$ C. Campañá and M. H. Müser, EPL 77, 38005 (2007).

${ }^{71}$ C. Campañá, M. H. Müser, and M. O. Robbins, J. Phys.: Condens. Matter 20, 354013 (2008)

${ }^{72}$ B. N. J. Persson, O. Albohr, G. Heinrich, and H. Ueba, J. Phys.: Condens. Matter 17, R1071 (2005).

${ }^{73}$ M. H. Müser, Phys. Rev. Lett. 100, 055504 (2008).

${ }^{74}$ C. Yang and B. N. J. Persson, J. Phys.: Condens. Matter 20, 215214 (2008).

${ }^{75}$ M. Enachescu, R. J. A. van den Oetelaar, R. W. Carpick, D. F. Ogletree, C. F. J. Flipse, and M. Salmeron, Phys. Rev. Lett. 81, 1877 (1998).

${ }^{76}$ M. Enachescu, R. J. A. van den Oetelaar, R. W. Carpick, D. F. Ogletree, C. F. J. Flipse, and M. Salmeron, Tribol. Lett. 7, 73 (1999).

${ }^{77}$ Y. Mo, M. H. Müser, and I. Szlufarska, Phys. Rev. B 80, 155438 
(2009).

${ }^{78}$ R. W. Carpick, D. F. Ogletree, and M. Salmeron, J. Colloid Interface Sci. 211, 395 (1999).

${ }^{79}$ According to continuum theories, Tabor's parameter (which can be related to the COS parameter $\alpha$ ), should be linear with $R^{1 / 3}$. While our data show the correct trend of $\alpha$ with $R$, it does not follow the predicted functional dependence. Given that only four tip radii $R$ have been considered in our study, we cannot quantify these deviations.

${ }^{80}$ K. Miura, N. Sasaki, and S. Kamiya, Phys. Rev. B 69, 075420 (2004).

${ }^{81}$ D. Dietzel, C. Ritter, T. Monninghoff, H. Fuchs, A. Schirmeisen, and U. D. Schwarz, Phys. Rev. Lett. 101, 125505 (2008). 